\title{
Ambient air pollutants increase the risk of Immunoglobulin E-mediated allergic diseases: a systematic review and meta-analysis
}

\author{
Hua Wang \\ Anhui Medical University School of Public Health \\ Xian-Bao Li \\ Anhui Medical University School of Public Health \\ Xiu-Jie Chu \\ Anhui Medical University School of Public Health \\ Nv-Wei Cao \\ Anhui Medical University School of Public Health \\ Hong Wu \\ Anhui Medical University School of Public Health \\ Rong-Gui Huang \\ Anhui Medical University School of Public Health \\ Bao-Zhu Li ( Ibz88730@163.com) \\ Anhui Medical University School of Public Health \\ Dong-Qing Ye \\ Anhui Medical University School of Public Health
}

\section{Research Article}

Keywords: Air pollutants, Systemic review, Eczema, Atopic dermatitis, Allergic rhinitis

Posted Date: January 21st, 2022

DOI: https://doi.org/10.21203/rs.3.rs-1213869/v1

License: @ (i) This work is licensed under a Creative Commons Attribution 4.0 International License. Read Full License 


\section{Abstract}

Immunoglobulin E (IgE)-mediated allergic diseases, including eczema, atopic dermatitis (AD) and allergic rhinitis (AR), have increased prevalence in recent decades. Recent studies have proved that environmental pollution might have correlations with IgE-mediated allergic diseases, but existing research findings were controversial. Thus, we performed a comprehensive meta-analysis from published observational studies to evaluate the risk of long-term and short-term exposed to air pollutants on the eczema, $\mathrm{AD}$ and $\mathrm{AR}$ in the population (per $10 \mu \mathrm{g} / \mathrm{m}^{3}$ increase in $\mathrm{PM}_{2.5}$ and $\mathrm{PM}_{10}$; per $1 \mathrm{ppb}$ increase in $\mathrm{SO}_{2}, \mathrm{NO}_{2}, \mathrm{CO}$ and $\mathrm{O}_{3}$ ). PubMed, Embase and Web of Science were searched to identify qualified literatures. Cochran $\mathrm{Q}$ test was used to assess heterogeneity and quantified with $P$ statistic. Pooled effects and the $95 \%$ confidence intervals (Cls) were used to evaluate outcome effects. A total of 55 articles were included in the study. The results showed that long-term and short-term exposed to $\mathrm{PM}_{10}$ increased the risk of eczema $\left(\mathrm{PM}_{10}, \mathrm{RR}_{\text {long }}=1.583,95 \% \mathrm{Cl}\right.$ : $1.328,1.888$; $\mathrm{RR}$ short $=1.006,95 \% \mathrm{Cl}$ : 1.003-1.008) and short-term exposed to $\mathrm{NO}_{2}\left(\mathrm{RR}_{\text {short }}=1.009,95 \% \mathrm{Cl}: 1.008-1.011\right)$ was associated with eczema. Short-term exposed to $\mathrm{SO}_{2}\left(\mathrm{RR}_{\text {short }}: 1.008\right.$, $95 \% \mathrm{Cl}: 1.001-1.015)$ was associated with the risk of $A D$. For $A R, P_{2.5}\left(R_{\text {long }}=1.058,95 \%\right.$ Cl: $\left.1.014-1.222\right)$ was harmful in long-term, and short-term exposed to $\mathrm{PM}_{10}\left(\mathrm{RR}_{\text {short }}: 1.028,95 \% \mathrm{Cl}: 1.008-1.049 ;\right)$ and $\mathrm{NO}_{2}\left(\mathrm{RR}_{\text {short }}: 1.018,95 \% \mathrm{Cl}: 1.007-1.029\right)$ were risk factors. The findings indicated that exposed to air pollutants might increase the risk of IgE-mediated allergic diseases. Further studies are warranted to illustrate potential mechanism for air pollutants and allergic diseases.

\section{Introduction}

Allergic diseases are inflammatory disorders that involve various types of cells and factors, including allergens, immunoglobulin (Ig)E, mast cells, basophils, cytokines, and soluble mediators. The etiological mechanisms of allergic diseases are complex. The incidence of allergic diseases has increased sharply with increasing industrialization and the accompanying changes to the environment and people's lifestyles. According to one study conducted by the World Allergy Organization and involved 30-nation/region, approximately 250 million (22\%) of the 1.2 billion people in those regions suffered from allergic diseases (Hu et al., 2018). Because of their high prevalence, these diseases pose a serious financial threat to affected households and consume substantial resources in socialized healthcare systems.

Allergic diseases can be divided into two categories, which are IgE mediated and non-IgE mediated. IgE-mediated allergies reactions are typically of rapid onset and symptoms range from mild to severe. IgE, an antibody class found only in mammals, has unique properties, and plays a central role in the development of acute allergic reactions and IgE-mediated allergic diseases. IgE-mediated allergic diseases, involving eczema, atopic dermatitis (AD) and allergic rhinitis (AR). Major risk factors for IgE mediated allergic diseases studied widely were genetics and immune functions. However, these traditional risk factors were not changed dramatically in recent decades. Therefore, traditional risk factors alone may not be sufficient to explain the massive rise in IgE mediated allergic diseases prevalence.

Are long-term or short-term air pollution associated with the development and prevalence of IgE-mediated allergic conditions? Numerous studies have attempted to answer this question, but no consensus has reached. For instance, Schnass et al. conducted a cohort study and concluded that traffic-related air pollution would increase the prevalence of eczema for elder (Schnass et al., 2018). However, Lopez et al. found that long-term air pollution has no adverse effect on adult eczema (Lopez et al., 2021). The same dispute can also be found in AR's research. Huang et al reported the prevalence of AR in children would increase when exposed to $\mathrm{PM}_{2.5}$ (Huang et al., 2019). But a cohort study established in Canada reported that PM2.5 did not increase the risk of AR for children (To et al., 2020). There are also meta-analyses tried to illustrate this question, and several important but inconsistent results have been received. In 2014 , a meta-analysis on allergy and sensitization found an association between air pollution exposure and childhood eczema, whereas another meta-analysis found no association between air pollution and eczema (Fuertes et al., 2020).

These contrary results might reveal the impact of different inclusion criteria, regions, and other factors on the generalization of the results. Usually, clinicians tend to interchangeably use the terms eczema and atopic dermatitis (AD). However, the term eczema is considered an ambiguous term and its meaning should not be considered synonymous with AD. In International Classification of Diseases (ICD), AD was only classified into the ICD-10 code L20, ICD-9 code 691 catalogue, whereas eczema could be classified into ICD-10 code L30. A diagnosis of AD is thought to confer a worse prognosis in terms of disease severity and the potential risk for developing other comorbid diseases of an atopic nature compared with receiving a diagnosis of eczema alone. Most of the existing meta-analyses did not consider the inclusion criteria of $A D$ and eczema and mixed these two diseases to conducted meta-analyses. Therefore, there is a need to divide these two diseases separately to conduct a meta-analysis to better understand the relationship between air pollution and IgE mediated allergic diseases.

To address the gap of inadequate knowledge of IgE-mediated allergic diseases and air pollutants, this systematic review and meta-analysis was conducted. Long-term and short-term effects of ambient six pollutants $\left(\mathrm{PM}_{2.5}, \mathrm{PM}_{10}, \mathrm{NO}_{2}, \mathrm{SO}_{2}, \mathrm{CO}, \mathrm{O}_{3}\right)$ on risks for IgE-mediated allergic diseases are comprehensive analyzed in this study.

\section{Material And Methods Search strategy}

Embase, PubMed and Web of science were searched to find relevant research concerning the association between air pollutants and diseases up to May 2021. Search terms included (1) "allergic rhinitis", "allergic respiratory diseases", "eczema", "eczematous dermatitides", "atopic dermatitis", "dermatitis atopic"; (2) "carbon monoxide", "sulfur dioxide", "particulate matter", "nitrogen dioxide", "ozone", "PM 2.5 ", "PM 10 ". Also, synonyms of diseases and particulate matters were searched using Medical Subjects Headings terms. The detailed search process was shown in supplementary Table S1. 


\section{Inclusion and exclusion criteria}

The included criteria were based on the population, exposure, comparator, and outcomes (PECO) framework. A framework was used to explore the association of air pollutants exposure with health outcomes. $\mathrm{P}$ refers to people who have IgE-mediated allergic diseases. $\mathrm{E}$ refers to air pollutants. $\mathrm{C}$ refers to incremental effect of per unit increased in concentration of air pollutants for diseases risk. O refers to the outcome of eczema, AR and AD (Morgan et al., 2018; Marx et al., 2021).

The inclusive criteria were as follows. (1) Articles should be epidemiologic studies focusing on the associations between the IgE-mediated allergic diseases with air pollutants exposure. (2) Eczema diagnosis was made according to ICD-10 code L30. ICD-10 code L20, and ICD-9 code 691 were used to classify AD. ICD-10 code J30 and ICD-9 code 477 were principles to detect AR. The classification of these three diseases could also be based on questionnaires of eczema or AD or AR. (3) Studies reported effect estimates (RR, OR, HR, PC) or data that could calculate the effect size. Animal studies, mechanism studies, reviews and meta-analyses, case reports, treatment effect evaluations and studies without original data were excluded. Studies focusing on the association between indoor air pollution and prenatal and allergic diseases were also excluded.

\section{Data extraction}

Endnote software (X9 version) was used to screen eligible literature. All articles were evaluated by two investigators. First, duplicated studies were removed. Then, two investigators (Wang $\mathrm{H}$ and Li XB) independently screened remaining studies to select eligible studies. When controversy existed, a third investigator was asked to discuss and resolve the disagreement.

For each included study, basic characters were extracted, including disease, first author, publication year, region, study design, sample size, number of cases, age, ICD, data sources of pollutants, term of exposure (short-term or long-term) (Ibrahim et al., 2021), mean concentration of pollutants and impact effect estimates. Investigators extracted information based on the following principle. A single pollutant model was used to find the effect of a pollutant, and a multipollutant model was utilized to explore the interactions of multiple pollutants on disease risk. If the study contained single pollutant and multi-pollutant models, the former would be chosen (Yang et al., 2018).

\section{Quality and risk bias assessment}

The Newcastle-Ottawa Scale (NOS) and the office of health assessment and translation (OHAT) tool were used to evaluate the quality of included literature. Among them, NOS was used to assess the reported quality of cohort, case-control, and cross-sectional studies (Lin et al., 2021a). NOS has eight items, and its score ranged from 0 to 9 . A study with a score higher than 7 was regarded as a high-quality study. A study with a score of 3 to 6 was intermediate quality. Otherwise, it was low quality. To the best of our knowledge, there is no effective scale to assess the quality of time series literature. Therefore, we adopted the quality scale used by Mustafic et al. This scale mainly evaluates three aspects: the validity of the outcome event, the assessment of air pollutant exposure and the adjustment of confounding factors. When the evaluated document score was 3-5 points, it could be considered high-quality (Mustafic et al., 2012). In a meta-analysis, OHAT tool was used to assess the risk of bias in each study (Zhang et al., 2021a).

\section{Data synthesis}

Due to inconsistent units of pollutant concentration in some literature, we standardized all effect sizes for every 10 ug/m $\mathrm{m}^{3}$ increase in $\mathrm{PM} \mathrm{M}_{2.5}$ and $\mathrm{PM} \mathrm{M}_{10}$ and 1 $\mathrm{ppb}$ increase in $\mathrm{NO}_{2}, \mathrm{SO}_{2}, \mathrm{CO}$ and $\mathrm{O}_{3}$ (Fan et al., 2020). The specific formulas were as follows: $1 \mathrm{ppm}=1000 \mathrm{ppb}, 1 \mathrm{ppb}=\mathrm{M} / 22.4$ (ug/m $\mathrm{m}^{3}$. $\mathrm{M}$ refers to the molecular weight of each air pollutant. Adjusted relative risk (RR), odds ratio (OR), risk ratio (HR) and percentage change (PC) were used to assess the risk of eczema, AR and AD (Ning et al., 2021). During data consolidation, PC was transformed into RR. The effect estimates (RR/OR/HR) were standardized. OR and HR were roughly regarded as RR, when outcome events were popular and the effect size was small (Chen et al., 2017). All the effect sizes were pooled by the standardized increment of environmental pollutant concentration. The standardized formulae of effect sizes were as follows:

$$
\begin{aligned}
& \mathrm{RR}_{(\text {standardized) }}=\mathrm{RR}_{\text {(original) }} \text { Increment(10)/Increment (original) } \\
& \mathrm{OR}_{(\text {standardized) }}=\mathrm{OR}_{\text {(original) }} \text { Increment(10)/Increment (original) } \\
& \mathrm{HR}_{(\text {standardized) }}=\mathrm{HR}_{\text {(original) }} \text { Increment(10)/Increment (original) }
\end{aligned}
$$

Cochran's Q-test and $R^{2}$ statistic were used to evaluate the heterogeneity between studies. If $P$ is greater than $50 \%$, the heterogeneity is high. Otherwise, the heterogeneity is low. If the $p$-value of the $Q$ test is less than 0.05 , a high heterogeneity is between the studies. Then the random effect model was chosen. The fixed effect model was applied if the heterogeneity was "medium or low". Funnel plots were used to represent the publication bias in studies (Bai et al., 2020). In addition, the pooled effect values were tested by performing subgroup analyses on ICD, age (<18 years old; $\geq 18$ years old; all ages), regional and study types for each pollutant (Chevalier et al., 2015). Limited by the number of available studies, sensitivity analyses were performed for studies that could be combined in each pollutant. All data analyses were realized by R packages "metafor" and "forestplot" in version 4.0.3.

\section{Results}

\section{Characteristics of included studies}

A total of 2478 articles were searched. After screening title and abstract, 150 articles were identified. After thoroughly reading the full text, a total of fifty-five articles were finally included. The process of literature screening was detailed in Figure 1. In four articles, two diseases were simultaneously discussed (Kim et al., 2016; Wang et al., 2016; Min et al., 2020; To et al., 2020). Therefore, included studies were as follows. There were seventeen eczema studies (six time- 
series, six cohorts, and five cross-sectional studies), thirty-one AR studies (nine time-series, nine cohorts, one case-control, and twelve cross-sectional studies), and eleven AD studies (four time-series, five cohorts, and two cross-sectional studies). The information extracted from the literature are shown in Table 1. According to the NOS scale and the OHAT tool, the qualities of the included articles were high. Scores of articles and details of risk bias assessment were listed in supplementary Table S2 (eczema), Table S3 (AD) and Table S4 (AR). 
Table 1

Characteristics of included studies

\begin{tabular}{|c|c|c|c|c|c|c|c|c|c|}
\hline Disease & $\begin{array}{l}\text { First } \\
\text { author/ } \\
\text { publication } \\
\text { year }\end{array}$ & Region & $\begin{array}{l}\text { Study } \\
\text { design }\end{array}$ & Sample size/Cases & $\begin{array}{l}\text { Age } \\
\text { (years) }\end{array}$ & ICD & $\begin{array}{l}\text { Data sources } \\
\text { of pollutants }\end{array}$ & Duration & $\begin{array}{l}\text { Mean } \\
\text { Concentration }\end{array}$ \\
\hline \multirow[t]{8}{*}{ Eczema } & $\begin{array}{l}\text { Brauer et } \\
\text { al (2007) }\end{array}$ & Netherlands & Cohort & $2571 /-$ & 4 & questionnaire & $\begin{array}{l}\text { monitoring } \\
\text { campaign }\end{array}$ & long-term & $\mathrm{PM}_{2.5}: 16.9 \mathrm{ug} / \mathrm{m}^{\prime}$ \\
\hline & & & & & & & & & $\mathrm{NO}_{2}: 25.2 \mathrm{ug} / \mathrm{m}^{3}$ \\
\hline & $\begin{array}{l}\text { Krämer et } \\
\text { al (2009) }\end{array}$ & Germany & Cohort & $2753 / 1741$ & $0-6$ & questionnaire & $\begin{array}{l}\text { monitoring } \\
\text { stations }\end{array}$ & long-term & $\mathrm{NO}_{2}: 23.7 \mathrm{ug} / \mathrm{m}^{3}$ \\
\hline & $\begin{array}{l}\text { Gehring et } \\
\text { al. (2010) }\end{array}$ & Netherlands & Cohort & $3184 /-$ & 8 & questionnaire & $\begin{array}{l}\text { land-use } \\
\text { regression } \\
\text { model }\end{array}$ & long-term & $\mathrm{PM}_{2.5}: 16.9(\mu \mathrm{g} / \mathrm{n}$ \\
\hline & $\begin{array}{l}\text { Aguilera et } \\
\text { al (2013) }\end{array}$ & Spanish & Cohort & $2199 / 460$ & $1-1.5$ & questionnaire & $\begin{array}{l}\text { Monitoring } \\
\text { stations }\end{array}$ & long-term & - \\
\hline & $\begin{array}{l}\text { Schnass } \\
\text { et.al. } \\
\text { (2018) }\end{array}$ & Germany & Cohort & $760 / 60$ & 73.5 & questionnaire & $\begin{array}{l}\text { monitoring } \\
\text { campaign }\end{array}$ & long-term & $\mathrm{PM}_{2.5}, 32.11 \mathrm{ug} / \mathrm{m}$ \\
\hline & $\begin{array}{l}\text { Lopez et.al } \\
\text { (2021) }\end{array}$ & Australian & Cohort & $3152 / 115$ & 53 & questionnaire & $\begin{array}{l}\text { monitoring } \\
\text { sites }\end{array}$ & long-term & $\mathrm{PM}_{2.5}: 6.4 \mathrm{ug} / \mathrm{m}^{3}$ \\
\hline & $\begin{array}{l}\text { Anderson } \\
\text { et al. } \\
\text { (2010) }\end{array}$ & Internationa- & $\begin{array}{l}\text { Cross- } \\
\text { sectional }\end{array}$ & $-/ 3086 /$ per center & $13-14$ & questionnaire & $\begin{array}{l}\text { monitoring } \\
\text { stations }\end{array}$ & short-term & $\mathrm{PM}_{10}: 34 \mathrm{ug} / \mathrm{m}^{3}$ \\
\hline \multirow[t]{5}{*}{ Disease } & First & Region & Study & Sample size/Cases & Age & ICD & Data sources & Duration & Mean \\
\hline & $\begin{array}{l}\text { author/ } \\
\text { publication } \\
\text { year }\end{array}$ & & & & & & of pollutants & & Concentration \\
\hline & $\begin{array}{l}\text { Liu et al. } \\
\text { (2016) }\end{array}$ & China & $\begin{array}{l}\text { Cross- } \\
\text { sectional }\end{array}$ & $3358 /-$ & $4-6$ & questionnaire & $\begin{array}{l}\text { Shanghai } \\
\text { Environmental } \\
\text { Monitoring } \\
\text { Center }\end{array}$ & long-term & $\begin{array}{l}\mathrm{PM}_{10}: 79.4 \mathrm{ug} / \mathrm{m}^{3} \\
\mathrm{NO}_{2}: 53.6 \mathrm{ug} / \mathrm{m}^{3} ; \mathrm{c}\end{array}$ \\
\hline & $\begin{array}{l}\text { Kathuria et } \\
\text { al. (2016) }\end{array}$ & U.S & $\begin{array}{l}\text { Cross- } \\
\text { sectional }\end{array}$ & $91642 / 11895$ & $0-17$ & questionnaire & $\begin{array}{l}\text { Environ } \\
\text { mental } \\
\text { Protection } \\
\text { Agency }\end{array}$ & short-term & $\begin{array}{l}\mathrm{PM}_{2.5}: 6.187 \mathrm{ug} / \mathrm{n} \\
\mathrm{PM}_{10}: 24.996 \mathrm{ug} / \mathrm{I}\end{array}$ \\
\hline & & & & & & & & & $\begin{array}{l}\mathrm{NO}_{2}, 12.851 \mathrm{ppm} ; \\
\mathrm{CO} 1.161 \mathrm{ppm} ; \mathrm{O}\end{array}$ \\
\hline
\end{tabular}

\begin{tabular}{|c|c|c|c|c|c|c|c|c|}
\hline $\begin{array}{l}\text { Deng et al. } \\
\text { (2019) }\end{array}$ & China & $\begin{array}{l}\text { Cross- } \\
\text { sectional }\end{array}$ & $3167 / 848$ & $3-6$ & questionnaire & $\begin{array}{l}\text { monitoring } \\
\text { stations }\end{array}$ & short-term & $\begin{array}{l}\mathrm{PM}_{2.5}: 72.11 \mathrm{ug} / \mathrm{n} \\
\mathrm{PM}_{10}: 115.58 \mathrm{ug} / \mathrm{I}\end{array}$ \\
\hline $\begin{array}{l}\text { Min et al. } \\
\text { (2020) }\end{array}$ & Korea & $\begin{array}{l}\text { Cross- } \\
\text { sectional }\end{array}$ & $14614 / 2323$ & $1-12$ & questionnaire & $\begin{array}{l}\text { monitoring } \\
\text { station }\end{array}$ & short-term & $\begin{array}{l}\mathrm{PM}_{2.5}: 25.13 \mu \mathrm{g} / \mathrm{m} \\
\mathrm{PM}_{10}: 49.36 \mu \mathrm{g} / \mathrm{m} \\
\mathrm{NO}_{2}: 35.6 \mu \mathrm{g} / \mathrm{m}^{3}\end{array}$ \\
\hline $\begin{array}{l}\text { Li et al. } \\
\text { (2016) }\end{array}$ & China & $\begin{array}{l}\text { Time- } \\
\text { series }\end{array}$ & $\begin{array}{l}-/ 510158 \\
\text { (outpatient visits) }\end{array}$ & - & ICD-10: L30.9 & $\begin{array}{l}\text { monitoring } \\
\text { station }\end{array}$ & short-term & $\begin{array}{l}\mathrm{PM}_{10}: 83 \mu \mathrm{g} / \mathrm{m}^{3} \\
\mathrm{NO}_{2}: 60 \mu \mathrm{g} / \mathrm{m}^{3} ; \mathrm{sc}\end{array}$ \\
\hline
\end{tabular}




\begin{tabular}{|c|c|c|c|c|c|c|c|c|c|}
\hline Disease & $\begin{array}{l}\text { First } \\
\text { author/ } \\
\text { publication } \\
\text { year }\end{array}$ & Region & $\begin{array}{l}\text { Study } \\
\text { design }\end{array}$ & Sample size/Cases & $\begin{array}{l}\text { Age } \\
\text { (years) }\end{array}$ & ICD & $\begin{array}{l}\text { Data sources } \\
\text { of pollutants }\end{array}$ & Duration & $\begin{array}{l}\text { Mean } \\
\text { Concentration }\end{array}$ \\
\hline \multirow[t]{6}{*}{ Disease } & $\begin{array}{l}\text { First } \\
\text { author/ } \\
\text { publication } \\
\text { year }\end{array}$ & Region & $\begin{array}{l}\text { Study } \\
\text { design }\end{array}$ & Sample size/Cases & $\begin{array}{l}\text { Age } \\
\text { (years) }\end{array}$ & ICD & $\begin{array}{l}\text { Data sources } \\
\text { of pollutants }\end{array}$ & Duration & $\begin{array}{l}\text { Mean } \\
\text { Concentration }\end{array}$ \\
\hline & $\begin{array}{l}\text { Li et al. } \\
\text { (2018) }\end{array}$ & China & $\begin{array}{l}\text { Time- } \\
\text { series }\end{array}$ & $\begin{array}{l}-/ 2305 \\
\text { (outpatient visits) }\end{array}$ & - & ICD-10: L30.9 & $\begin{array}{l}\text { monitoring } \\
\text { station }\end{array}$ & short-term & $\begin{array}{l}\mathrm{PM}_{10}, 119.6 \mu \mathrm{g} / \mathrm{m} \\
\mathrm{NO}_{2}: 55.2 \mu \mathrm{g} / \mathrm{m}^{3} ;\end{array}$ \\
\hline & $\begin{array}{l}\text { Wang et al. } \\
(2019)\end{array}$ & China & $\begin{array}{l}\text { Time- } \\
\text { series }\end{array}$ & $\begin{array}{l}-/ 2585 \\
\text { (outpatient visits) }\end{array}$ & $\geq 18$ & ICD-10: L30.9 & $\begin{array}{l}\text { monitoring } \\
\text { station }\end{array}$ & short-term & $\mathrm{PM}_{2.5}: 101.2 \mu \mathrm{g} / \mathrm{m}$ \\
\hline & $\begin{array}{l}\text { Guo et.al } \\
\text { (2019) }\end{array}$ & China & $\begin{array}{l}\text { Time- } \\
\text { series }\end{array}$ & $\begin{array}{l}\text {-/157595 (outpatient } \\
\text { visits) }\end{array}$ & - & $\begin{array}{l}\text { ICD-10: L20- } \\
\text { L30 }\end{array}$ & $\begin{array}{l}\text { Beijing } \\
\text { Municipal } \\
\text { Environmental } \\
\text { Monitoring } \\
\text { Center }\end{array}$ & short-term & $\begin{array}{l}\mathrm{PM}_{2.5}: 87.4 \mathrm{ug} / \mathrm{m}^{\prime} \\
\mathrm{PM}_{10}: 116.6 \mathrm{ug} / \mathrm{m} \\
\mathrm{NO}_{2}: 53.1 \mathrm{ug} / \mathrm{m}^{3} \\
\mathrm{SO}_{2}: 27.1 \mathrm{ug} / \mathrm{m}^{3}\end{array}$ \\
\hline & $\begin{array}{l}\text { Karagün et } \\
\text { al. (2021) }\end{array}$ & Turkish & $\begin{array}{l}\text { Time- } \\
\text { series }\end{array}$ & $\begin{array}{l}-/ 27549 \text { (outpatient } \\
\text { visits) }\end{array}$ & - & $\begin{array}{l}\text { ICD-10:L-20, } \\
\text { L-25, and L- } \\
30\end{array}$ & $\begin{array}{l}\text { monitoring } \\
\text { station }\end{array}$ & short-term & $\begin{array}{l}\mathrm{PM}_{10}: 82.8 \mu \mathrm{g} / \mathrm{m}^{3} \\
\mathrm{SO}_{2}, 7.6 \mu \mathrm{g} / \mathrm{m}^{3}\end{array}$ \\
\hline & $\begin{array}{l}\text { Zhang et } \\
\text { al. } \\
(2021)\end{array}$ & China & $\begin{array}{l}\text { Time- } \\
\text { series }\end{array}$ & $\begin{array}{l}\text {-/293340 (outpatie-nt } \\
\text { visits) }\end{array}$ & - & $\begin{array}{l}\text { ICD-10: } \\
\text { L30.902 }\end{array}$ & $\begin{array}{l}\text { monitoring } \\
\text { station }\end{array}$ & short-term & - \\
\hline Disease & $\begin{array}{l}\text { First } \\
\text { author/ } \\
\text { publication } \\
\text { year }\end{array}$ & Region & $\begin{array}{l}\text { Study } \\
\text { design }\end{array}$ & Sample size/Cases & $\begin{array}{l}\text { Age } \\
\text { (years) }\end{array}$ & ICD & $\begin{array}{l}\text { Data sources } \\
\text { of pollutants }\end{array}$ & Duration & $\begin{array}{l}\text { Mean } \\
\text { Concentration }\end{array}$ \\
\hline$A D$ & $\begin{array}{l}\text { Wang et al. } \\
\text { (2015) }\end{array}$ & Taipei & Cohort & $2661 / 383$ & 5.5 & questionnaire & $\begin{array}{l}\text { monitoring } \\
\text { station }\end{array}$ & long-term & $\begin{array}{l}\mathrm{PM}_{2.5}, 29.07 \mathrm{ug} / \mathrm{m} \\
\mathrm{PM}_{10}: 48.32 \mathrm{ug} / \mathrm{m} \\
\mathrm{SO}_{2}: 6.46 \mathrm{ppb} ; \\
\mathrm{CO}: 0.63 \mathrm{ppm} \\
\mathrm{O}_{3}: 27.62 \mathrm{ppb} ;\end{array}$ \\
\hline & $\begin{array}{l}\text { Hüls et al. } \\
\text { (2018) }\end{array}$ & Canada & Cohort & $5132 / 440$ & $7-8$ & questionnaire & $\begin{array}{l}\text { land-use } \\
\text { regression } \\
\text { models }\end{array}$ & long-term & $\mathrm{NO}_{2}$ \\
\hline & $\begin{array}{l}\text { Belugina et } \\
\text { al (2018) }\end{array}$ & Minsk & Cohort & $\begin{array}{l}\text {-/12-335 cases per } \\
100,000 \text { person-year }\end{array}$ & $0-2$ & $\begin{array}{l}\text { ICD- } \\
\text { 10:L20.80 }\end{array}$ & $\begin{array}{l}\text { National } \\
\text { Academy of } \\
\text { Science of } \\
\text { Belarus }\end{array}$ & long-term & $\begin{array}{l}\mathrm{PM}_{10}: 27.94 \% ; \mathrm{NC} \\
\mathrm{CO}: 584.4 \mathrm{ug} / \mathrm{m}^{3} ; \\
\mathrm{O}_{3}: 31.19 \mathrm{ppb} .\end{array}$ \\
\hline
\end{tabular}

\begin{tabular}{|c|c|c|c|c|c|c|c|c|}
\hline $\begin{array}{l}\text { To et.al } \\
(2020)\end{array}$ & Canada & Cohort & $1286 / 958$ & 3 & $\begin{array}{l}\text { ICD-9: } 691.8 \\
\text { ICD-10: L20 }\end{array}$ & monitors & long-term & $\begin{array}{l}\mathrm{PM}_{2.5}: 10.88 \mathrm{ug} / \mathrm{n} \\
\mathrm{NO}_{2}: 26.14 \mathrm{ug} / \mathrm{m}^{3}\end{array}$ \\
\hline
\end{tabular}




\begin{tabular}{|c|c|c|c|c|c|c|c|c|c|}
\hline Disease & $\begin{array}{l}\text { First } \\
\text { author/ } \\
\text { publication } \\
\text { year }\end{array}$ & Region & $\begin{array}{l}\text { Study } \\
\text { design }\end{array}$ & Sample size/Cases & $\begin{array}{l}\text { Age } \\
\text { (years) }\end{array}$ & ICD & $\begin{array}{l}\text { Data sources } \\
\text { of pollutants }\end{array}$ & Duration & $\begin{array}{l}\text { Mean } \\
\text { Concentration }\end{array}$ \\
\hline \multirow[t]{5}{*}{ Disease } & $\begin{array}{l}\text { First } \\
\text { author/ } \\
\text { publication } \\
\text { year }\end{array}$ & Region & $\begin{array}{l}\text { Study } \\
\text { design }\end{array}$ & Sample size/Cases & $\begin{array}{l}\text { Age } \\
\text { (years) }\end{array}$ & ICD & $\begin{array}{l}\text { Data sources } \\
\text { of pollutants }\end{array}$ & Duration & $\begin{array}{l}\text { Mean } \\
\text { Concentration }\end{array}$ \\
\hline & $\begin{array}{l}\text { Park et al. } \\
(2021)\end{array}$ & Korea & Cohort & 209168/3203 & - & ICD-10:L20 & $\begin{array}{l}\text { Korean } \\
\text { Department of } \\
\text { Environmental } \\
\text { Protection }\end{array}$ & long-term & - \\
\hline & $\begin{array}{l}\text { Kim et al. } \\
\text { (2016) }\end{array}$ & Korea & $\begin{array}{l}\text { Cross- } \\
\text { sectional }\end{array}$ & $1828 / 669$ & $6-7$ & questionnaire & $\begin{array}{l}\text { monitoring } \\
\text { sites }\end{array}$ & long-term & $\begin{array}{l}\mathrm{PM}_{10}: 58.8 \mu \mathrm{g} / \mathrm{m}^{3} \\
\mathrm{NO}_{2}: 29.7 \mathrm{ppb} \\
\mathrm{SO}_{2}: 5.2 \mathrm{ppb} ; \mathrm{CO}: \epsilon \\
\mathrm{O}_{3}: 30.7 \mathrm{ppb}\end{array}$ \\
\hline & $\begin{array}{l}\text { Tang et al. } \\
\text { (2017) }\end{array}$ & China & $\begin{array}{l}\text { Cross- } \\
\text { sectional }\end{array}$ & $6115 / 1023$ & $\geq 20$ & ICD-9: 691 & $\begin{array}{l}\text { Environmental } \\
\text { Protection } \\
\text { Agency } \\
\text { monitoring } \\
\text { stations }\end{array}$ & long-term & $\begin{array}{l}\mathrm{PM}_{2.5}: 33.6 \mu \mathrm{g} / \mathrm{m}^{\prime} \\
\mathrm{PM}_{10}: 56.3 \mu \mathrm{g} / \mathrm{m}^{3} \\
\mathrm{NO}_{2}: 18.6 \mathrm{ppb} ; \\
\mathrm{SO}_{2}: 4 \mathrm{ppb} ; \\
\mathrm{CO}: 0.5 \mathrm{ppb} ; \\
\mathrm{O}_{3}: 27.9 \mathrm{ppb}\end{array}$ \\
\hline & $\begin{array}{l}\text { Lee et al. } \\
\text { (2010) }\end{array}$ & Korea & $\begin{array}{l}\text { Time- } \\
\text { series }\end{array}$ & $\begin{array}{l}-/ 183+29 \\
\text { (daily } \\
\text { hospitaladmission) }\end{array}$ & $<15$ & ICD-10:L20 & $\begin{array}{l}\text { monitoring } \\
\text { station }\end{array}$ & short-term & $\begin{array}{l}\text { seoul : } \mathrm{O}_{3}, 26.09 \mathrm{pl} \\
\text { ulsan: } \mathrm{O}_{3}, 32.05 \mathrm{pr}\end{array}$ \\
\hline \multirow[t]{4}{*}{ Disease } & $\begin{array}{l}\text { First } \\
\text { author/ } \\
\text { publication } \\
\text { year }\end{array}$ & Region & $\begin{array}{l}\text { Study } \\
\text { design }\end{array}$ & Sample size/Cases & $\begin{array}{l}\text { Age } \\
\text { (years) }\end{array}$ & ICD & $\begin{array}{l}\text { Data sources } \\
\text { of pollutants }\end{array}$ & Duration & $\begin{array}{l}\text { Mean } \\
\text { Concentration }\end{array}$ \\
\hline & $\begin{array}{l}\text { Kim et al. } \\
(2017)\end{array}$ & Korea & $\begin{array}{l}\text { Time- } \\
\text { series }\end{array}$ & $-/ 117$ & $\begin{array}{l}2.0 \pm \\
1.6\end{array}$ & questionnaire & $\begin{array}{l}\text { National } \\
\text { Institute of } \\
\text { Environmental } \\
\text { Research }\end{array}$ & short-term & $\begin{array}{l}\mathrm{PM}_{10}: 45.2 \mathrm{ug} / \mathrm{m}^{3} \\
\mathrm{NO}_{2}: 32.4 \mathrm{ppb} ; \\
\mathrm{O}_{3}: 38.1 \mathrm{ppb}\end{array}$ \\
\hline & $\begin{array}{l}\text { Guo et al. } \\
\text { (2019) }\end{array}$ & China & $\begin{array}{l}\text { Time- } \\
\text { series }\end{array}$ & $\begin{array}{l}64987 \\
\text { (outpatie-nt visits) }\end{array}$ & - & ICD-10: L20. & $\begin{array}{l}\text { Monitoring } \\
\text { stations }\end{array}$ & short-term & $\begin{array}{l}\mathrm{PM}_{10}: 110.5 \mathrm{ug} / \mathrm{m} \\
\mathrm{PM}_{2.5}: 79.7 \mathrm{ug} / \mathrm{m}^{\prime} \\
\mathrm{SO}_{2}: 16.9 \mathrm{ug} / \mathrm{m}^{3}\end{array}$ \\
\hline & $\begin{array}{l}\text { Baek et al. } \\
(2021)\end{array}$ & Korea & $\begin{array}{l}\text { Time- } \\
\text { series }\end{array}$ & $\begin{array}{l}\text {-/513870(medical } \\
\text { care visits) }\end{array}$ & - & $\begin{array}{l}\text { ICD-10:L20.8, } \\
\text { L20.9 }\end{array}$ & $\begin{array}{l}\text { monitoring } \\
\text { station }\end{array}$ & short-term & - \\
\hline AR & $\begin{array}{l}\text { Kim et al. } \\
(2011)\end{array}$ & Korea & Cohort & $1340 /-$ & 6.84 & questionnaire & $\begin{array}{l}\text { monitoring } \\
\text { station }\end{array}$ & long-term & $\mathrm{O}_{3}: 37.93 \mu \mathrm{g} / \mathrm{m}^{3}$ \\
\hline
\end{tabular}




\begin{tabular}{|c|c|c|c|c|c|c|c|c|c|}
\hline Disease & $\begin{array}{l}\text { First } \\
\text { author/ } \\
\text { publication } \\
\text { year }\end{array}$ & Region & $\begin{array}{l}\text { Study } \\
\text { design }\end{array}$ & Sample size/Cases & $\begin{array}{l}\text { Age } \\
\text { (years) }\end{array}$ & ICD & $\begin{array}{l}\text { Data sources } \\
\text { of pollutants }\end{array}$ & Duration & $\begin{array}{l}\text { Mean } \\
\text { Concentration }\end{array}$ \\
\hline \multirow[t]{7}{*}{ Disease } & $\begin{array}{l}\text { First } \\
\text { author/ } \\
\text { publication } \\
\text { year }\end{array}$ & Region & $\begin{array}{l}\text { Study } \\
\text { design }\end{array}$ & Sample size/Cases & $\begin{array}{l}\text { Age } \\
\text { (years) }\end{array}$ & ICD & $\begin{array}{l}\text { Data sources } \\
\text { of pollutants }\end{array}$ & Duration & $\begin{array}{l}\text { Mean } \\
\text { Concentration }\end{array}$ \\
\hline & $\begin{array}{l}\text { Fuertes et } \\
\text { al. (2013) }\end{array}$ & Canada & Cohort & $10027 / 4736$ & 7 or 8 & questionnaire & $\begin{array}{l}\text { land-use } \\
\text { regres sion } \\
\text { modeling }\end{array}$ & long-term & $\mathrm{PM}_{2.5}, \mathrm{NO}_{2}, \mathrm{O}_{3}$ \\
\hline & $\begin{array}{l}\text { Fuertes et } \\
\text { al. (2013) }\end{array}$ & Germany & Cohort & $4623 / 460$ & 10 & questionnaire & $\begin{array}{l}\text { land-use } \\
\text { regression } \\
\text { models }\end{array}$ & long-term & $\begin{array}{l}\mathrm{PM}_{2.5}: 15.3 \mu \mathrm{g} / \mathrm{m}^{\prime} \\
\mathrm{NO}_{2}: 22.4 \mu \mathrm{g} / \mathrm{m}^{3} \\
\mathrm{O}_{3}: 42.5 \mu \mathrm{g} / \mathrm{m}^{3}\end{array}$ \\
\hline & $\begin{array}{l}\text { Wang et al. } \\
(2015)\end{array}$ & Taipei & Cohort & $2661 / 798$ & 5.5 & questionnaire & $\begin{array}{l}\text { monitoring } \\
\text { station }\end{array}$ & long-term & $\begin{array}{l}\mathrm{PM}_{2.5}, 29.07 \mathrm{ug} / \mathrm{m} \\
\mathrm{PM}_{10}: 48.32 \mathrm{ug} / \mathrm{m} \\
\mathrm{SO}_{2}: 6.46 \mathrm{ppb} ; \\
\mathrm{CO}: 0.63 \mathrm{ppm} \\
\mathrm{O}_{3}: 27.62 \mathrm{ppb} ;\end{array}$ \\
\hline & $\begin{array}{l}\text { Chung et } \\
\text { al. } \\
(2016)\end{array}$ & China & Cohort & $9960 / 1088$ & $0-6$ & $\begin{array}{l}\text { ICD-9- } \\
\text { CM:477.0, } \\
477.1,477.2 \\
477.8,477.9\end{array}$ & $\begin{array}{l}\text { Environmental } \\
\text { monitoring } \\
\text { sites }\end{array}$ & long-term & $\begin{array}{l}\mathrm{PM}_{10}, 56.8 \mu \mathrm{g} / \mathrm{m}^{3} \\
\mathrm{SO}_{2}, 4.81 \mathrm{ppb} ; \\
\mathrm{CO}, 561 \mathrm{ppb} ; \\
\mathrm{O}_{3}, 27.9 \mathrm{ppb}\end{array}$ \\
\hline & $\begin{array}{l}\text { Burte et al. } \\
(2018)\end{array}$ & Europe & Cohort & $1533 / 394$ & 42.7 & questionnaire & $\begin{array}{l}\text { monitoring } \\
\text { station }\end{array}$ & long-term & - \\
\hline & $\begin{array}{l}\text { To et al. } \\
\text { (2020) }\end{array}$ & Canada & Cohort & $1286 / 511$ & 3 & $\begin{array}{l}\text { ICD-9: } \\
\text { 477;ICD-10: } \\
\text { J301-J304 }\end{array}$ & monitors & long-term & $\begin{array}{l}\mathrm{PM}_{2.5}: 10.88 \mathrm{mg} / \mathrm{r} \\
\mathrm{NO}_{2}: 26.14 \mathrm{ppb} \\
\mathrm{O}_{3}: 43.72 \mathrm{ppb}\end{array}$ \\
\hline \multirow[t]{4}{*}{ Disease } & $\begin{array}{l}\text { First } \\
\text { author/ } \\
\text { publication } \\
\text { year }\end{array}$ & Region & $\begin{array}{l}\text { Study } \\
\text { design }\end{array}$ & Sample size/Cases & $\begin{array}{l}\text { Age } \\
\text { (years) }\end{array}$ & ICD & $\begin{array}{l}\text { Data sources } \\
\text { of pollutants }\end{array}$ & Duration & $\begin{array}{l}\text { Mean } \\
\text { Concentration }\end{array}$ \\
\hline & $\begin{array}{l}\text { Lin et al. } \\
(2021)\end{array}$ & China & Cohort & $140911 / 47276$ & 1 & $\begin{array}{l}\text { ICD-9: } \\
477.0,477.1 \\
477.2,477.8 \\
477.9\end{array}$ & $\begin{array}{l}\text { novel satellite- } \\
\text { based hybrid } \\
\text { model }\end{array}$ & long-term & $\mathrm{PM}_{2.5}: 33.84 \mu \mathrm{g} / \mathrm{n}$ \\
\hline & $\begin{array}{l}\text { Kim et al. } \\
\text { (2021) }\end{array}$ & Korea & Cohort & $3592 / 995$ & 9.08 & questionnaire & $\begin{array}{l}\text { national } \\
\text { monitoring } \\
\text { sites }\end{array}$ & long-term & $\begin{array}{l}\mathrm{PM}_{10}: 40.3 \mathrm{ug} / \mathrm{m}^{3} \\
\mathrm{SO}_{2}: 5.4 \mathrm{ppb} ; \mathrm{CO}: 5 \\
\mathrm{O}_{3}: 42.5 \mathrm{ppb}\end{array}$ \\
\hline & $\begin{array}{l}\text { de Marco } \\
\text { et al. } \\
\text { (2002) }\end{array}$ & Italy & $\begin{array}{l}\text { Cross- } \\
\text { sectional }\end{array}$ & $18873 / 3529$ & 33.1 & questionnaire & $\begin{array}{l}\text { monitoring } \\
\text { sites }\end{array}$ & long-term & $\mathrm{NO}_{2}: 31.46 \mu \mathrm{g} / \mathrm{m}^{3}$ \\
\hline
\end{tabular}




\begin{tabular}{|c|c|c|c|c|c|c|c|c|c|}
\hline Disease & $\begin{array}{l}\text { First } \\
\text { author/ } \\
\text { publication } \\
\text { year }\end{array}$ & Region & $\begin{array}{l}\text { Study } \\
\text { design }\end{array}$ & Sample size/Cases & $\begin{array}{l}\text { Age } \\
\text { (years) }\end{array}$ & ICD & $\begin{array}{l}\text { Data sources } \\
\text { of pollutants }\end{array}$ & Duration & $\begin{array}{l}\text { Mean } \\
\text { Concentration }\end{array}$ \\
\hline & $\begin{array}{l}\text { Hwang et } \\
\text { al. (2006) }\end{array}$ & China & $\begin{array}{l}\text { Cross- } \\
\text { sectional }\end{array}$ & $32143 / 8202$ & $6-15$ & questionnaire & $\begin{array}{l}\text { Environmental } \\
\text { Protection } \\
\text { Agency air- } \\
\text { monitoring } \\
\text { station. }\end{array}$ & long-term & $\begin{array}{l}\mathrm{PM}_{10}: 55.58 \mathrm{ug} / \mathrm{m} \\
\mathrm{SO}_{2}: 3.53 \mathrm{ppb} ; \\
\mathrm{CO}: 664 \mathrm{ppb} ; \\
\mathrm{O}_{3}: 23.14 \mathrm{ppb}\end{array}$ \\
\hline & $\begin{array}{l}\text { Arnedo- } \\
\text { Pena et al. } \\
\text { (2009) }\end{array}$ & Spain & $\begin{array}{l}\text { Cross- } \\
\text { sectional }\end{array}$ & $20455 /-$ & $6-7$ & questionnaire & $\begin{array}{l}\text { Pollutant } \\
\text { detection } \\
\text { systems of } \\
\text { centers }\end{array}$ & long-term & $\begin{array}{l}\mathrm{NO}_{2}: 40.4 \mathrm{ug} / \mathrm{m}^{3} \\
\mathrm{CO}: 0.8 \mathrm{ug} / \mathrm{m}^{3}\end{array}$ \\
\hline \multirow[t]{6}{*}{ Disease } & $\begin{array}{l}\text { First } \\
\text { author/ } \\
\text { publication } \\
\text { year }\end{array}$ & Region & $\begin{array}{l}\text { Study } \\
\text { design }\end{array}$ & Sample size/Cases & $\begin{array}{l}\text { Age } \\
\text { (years) }\end{array}$ & ICD & $\begin{array}{l}\text { Data sources } \\
\text { of pollutants }\end{array}$ & Duration & $\begin{array}{l}\text { Mean } \\
\text { Concentration }\end{array}$ \\
\hline & $\begin{array}{l}\text { Lu et al. } \\
\text { (2013) }\end{array}$ & China & $\begin{array}{l}\text { Cross- } \\
\text { sectional }\end{array}$ & $2159 / 182$ & $3-6$ & questionnaire & $\begin{array}{l}\text { Environmental } \\
\text { Protection } \\
\text { Agency }\end{array}$ & long-term & $\mathrm{PM}_{10} ; \mathrm{SO}_{2} ; \mathrm{NO}_{2}$ \\
\hline & $\begin{array}{l}\text { Wood et al. } \\
\text { (2015) }\end{array}$ & London & $\begin{array}{l}\text { Cross- } \\
\text { sectional }\end{array}$ & $1808 / 242$ & $8-9$ & questionnaire & $\begin{array}{l}\text { dispersion } \\
\text { models }\end{array}$ & short-term & $\begin{array}{l}\mathrm{PM}_{2.5}: 13.7 \mu \mathrm{g} / \mathrm{m}^{\prime} \\
\mathrm{PM}_{10}: 23.4 \mu \mathrm{g} / \mathrm{m}^{3} \\
\mathrm{NO}_{2}: 43.5 \mu \mathrm{g} / \mathrm{m}^{3}\end{array}$ \\
\hline & $\begin{array}{l}\text { Kim et al. } \\
(2016)\end{array}$ & Korea & $\begin{array}{l}\text { Cross- } \\
\text { sectional }\end{array}$ & $1828 / 673$ & $6-7$ & questionnaire & $\begin{array}{l}\text { monitoring } \\
\text { sites }\end{array}$ & long-term & $\begin{array}{l}\mathrm{PM}_{10}: 58.8 \mu \mathrm{g} / \mathrm{m} \\
\mathrm{SO}_{2}: 5.2 \mathrm{ppb} ; \\
\mathrm{CO}: 6.5(100 \mathrm{ppb}) \\
\mathrm{O}_{3}: 30.7 \mathrm{ppb} .\end{array}$ \\
\hline & $\begin{array}{l}\text { Chen et al. } \\
(2016)\end{array}$ & China & $\begin{array}{l}\text { Time- } \\
\text { series }\end{array}$ & $-/ 19370$ & $2-15$ & $\begin{array}{l}\text { experienced } \\
\text { physicians } \\
\text { diagnosed }\end{array}$ & $\begin{array}{l}\text { Shanghai } \\
\text { Environmental } \\
\text { Bureau }\end{array}$ & short-term & $\begin{array}{l}\mathrm{SO}_{2}: 39.63 \mu \mathrm{g} / \mathrm{m}^{3} \\
\mathrm{O}_{3}: 43.22 \mu \mathrm{g} / \mathrm{m}^{3}\end{array}$ \\
\hline & $\begin{array}{l}\text { Jo et al. } \\
\text { (2017) }\end{array}$ & Korea & $\begin{array}{l}\text { Cross- } \\
\text { sectional }\end{array}$ & $\begin{array}{l}\text {-/4.4(daily } \\
\text { admission) }\end{array}$ & - & ICD-10: J30 & $\begin{array}{l}\text { monitoring } \\
\text { stations }\end{array}$ & short-term & $\mathrm{PM}_{2.5}: 24.2 \mu \mathrm{g} / \mathrm{m}^{\prime}$ \\
\hline \multirow[t]{4}{*}{ Disease } & $\begin{array}{l}\text { First } \\
\text { author/ } \\
\text { publication } \\
\text { year }\end{array}$ & Region & $\begin{array}{l}\text { Study } \\
\text { design }\end{array}$ & Sample size/Cases & $\begin{array}{l}\text { Age } \\
\text { (years) }\end{array}$ & ICD & $\begin{array}{l}\text { Data sources } \\
\text { of pollutants }\end{array}$ & Duration & $\begin{array}{l}\text { Mean } \\
\text { Concentration }\end{array}$ \\
\hline & $\begin{array}{l}\text { Chen et al. } \\
(2018)\end{array}$ & China & $\begin{array}{l}\text { Cross- } \\
\text { sectional }\end{array}$ & $30756 / 204$ & 4.6 & questionnaire & $\begin{array}{l}\text { Global Burden } \\
\text { of Disease }\end{array}$ & long-term & $\mathrm{PM}_{2.5}: 64 \mu \mathrm{g} / \mathrm{m}^{3}$ \\
\hline & $\begin{array}{l}\text { Liu et al. } \\
\text { (2019) }\end{array}$ & China & $\begin{array}{l}\text { Cross- } \\
\text { sectional }\end{array}$ & $56137 / 5395$ & 10 & questionnaire & $\begin{array}{l}\text { monitoring } \\
\text { stations }\end{array}$ & short-term & $\begin{array}{l}\mathrm{PM}_{2.5}: 55.08 \mu \mathrm{g} / \mathrm{m} \\
\mathrm{PM}_{10}: 98.75 \mu \mathrm{g} / \mathrm{m} \\
\mathrm{NO}_{2}: 35.43 \mu \mathrm{g} / \mathrm{m}^{3}\end{array}$ \\
\hline & $\begin{array}{l}\text { Min et al. } \\
(2020)\end{array}$ & Korea & $\begin{array}{l}\text { Cross- } \\
\text { sectional }\end{array}$ & $14614 / 5286$ & $1-12$ & questionnaire & $\begin{array}{l}\text { monitoring } \\
\text { station }\end{array}$ & $\begin{array}{l}\text { dispersion } \\
\text { models }\end{array}$ & $\begin{array}{l}\mathrm{PM}_{2.5}: 25.13 \mu \mathrm{g} / \mathrm{m} \\
\mathrm{PM}_{10}: 49.36 \mu \mathrm{g} / \mathrm{m} \\
\mathrm{NO}_{2}: 35.6 \mu \mathrm{g} / \mathrm{m}^{3}\end{array}$ \\
\hline
\end{tabular}




\begin{tabular}{|c|c|c|c|c|c|c|c|c|c|}
\hline Disease & $\begin{array}{l}\text { First } \\
\text { author/ } \\
\text { publication } \\
\text { year }\end{array}$ & Region & $\begin{array}{l}\text { Study } \\
\text { design }\end{array}$ & Sample size/Cases & $\begin{array}{l}\text { Age } \\
\text { (years) }\end{array}$ & ICD & $\begin{array}{l}\text { Data sources } \\
\text { of pollutants }\end{array}$ & Duration & $\begin{array}{l}\text { Mean } \\
\text { Concentration }\end{array}$ \\
\hline & $\begin{array}{l}\text { Wang et al. } \\
(2020)\end{array}$ & China & $\begin{array}{l}\text { Cross- } \\
\text { sectional }\end{array}$ & $40279 / 2658$ & - & questionnaire & $\begin{array}{l}\text { National } \\
\text { Bureau of } \\
\text { Statistics }\end{array}$ & short-term & $\mathrm{PM}_{10}, \mathrm{NO}_{2}$ \\
\hline & $\begin{array}{l}\text { Hao et al. } \\
(2021)\end{array}$ & China & $\begin{array}{l}\text { Case- } \\
\text { Control }\end{array}$ & $3047 / 194$ & $2-4$ & questionnaire & $\begin{array}{l}\text { monitoring } \\
\text { statio }\end{array}$ & long-term & $\begin{array}{l}\mathrm{PM}_{10}: 88 \mathrm{ug} / \mathrm{m}^{3} ; \mathrm{r} \\
\mathrm{SO}_{2}: 26 \mathrm{ug} / \mathrm{m}^{3} ; \mathrm{C} \\
\mathrm{O}_{3}: 92 \mathrm{ug} / \mathrm{m}^{3}\end{array}$ \\
\hline & $\begin{array}{l}\text { Zhou et al. } \\
(2021)\end{array}$ & China & $\begin{array}{l}\text { Cross- } \\
\text { sectional }\end{array}$ & $59754 / 3186$ & 10 & questionnaire & $\begin{array}{l}\text { satellite-based } \\
\text { random forest } \\
\text { approach }\end{array}$ & long-term & $\mathrm{O}_{3}: 89.39 \mu \mathrm{g} / \mathrm{m}^{3}$ \\
\hline Disease & $\begin{array}{l}\text { First } \\
\text { author/ } \\
\text { publication } \\
\text { year }\end{array}$ & Region & $\begin{array}{l}\text { Study } \\
\text { design }\end{array}$ & Sample size/Cases & $\begin{array}{l}\text { Age } \\
\text { (years) }\end{array}$ & ICD & $\begin{array}{l}\text { Data sources } \\
\text { of pollutants }\end{array}$ & Duration & $\begin{array}{l}\text { Mean } \\
\text { Concentration }\end{array}$ \\
\hline & $\begin{array}{l}\text { Tecer et al. } \\
(2008)\end{array}$ & Zonguldak & $\begin{array}{l}\text { Time- } \\
\text { series }\end{array}$ & -/424 admissions & $0-14$ & $\begin{array}{l}\text { ICD-9: } 470- \\
478\end{array}$ & $\begin{array}{l}\text { Anderson } \\
\text { automatic } \\
\text { dichotomous } \\
\text { sampler }\end{array}$ & short-term & $\mathrm{PM}_{2.5}: 29.1 \mu \mathrm{g} / \mathrm{m}^{i}$ \\
\hline & $\begin{array}{l}\text { Zhang et } \\
\text { al. } \\
(2011)\end{array}$ & China & $\begin{array}{l}\text { Time- } \\
\text { series }\end{array}$ & -/1506(outpatien) & $\geq 20$ & questionnaire & $\begin{array}{l}\text { Beijing } \\
\text { Municipal } \\
\text { Environmental } \\
\text { Protection } \\
\text { Monitoring } \\
\text { Center }\end{array}$ & short-term & $\begin{array}{l}\mathrm{PM}_{10}: 116.092 \mu \mathrm{g} \\
\mathrm{SO}_{2}: 44.052 \mu \mathrm{g} / \mathrm{m}\end{array}$ \\
\hline & $\begin{array}{l}\text { Chen et al. } \\
(2016)\end{array}$ & China & $\begin{array}{l}\text { Time- } \\
\text { series }\end{array}$ & -/124773(clinicvisits) & - & ICD-9:477 & $\begin{array}{l}\text { monitoring } \\
\text { stations }\end{array}$ & short-term & $\begin{array}{l}\mathrm{PM}_{10}: 45.79 \mu \mathrm{g} / \mathrm{m} \\
\mathrm{SO}_{2}: 3.51 \mathrm{ppb} ; \\
\mathrm{CO}: 0.62 \mathrm{ppm} ; \\
\mathrm{O}_{3}: 23.77 \mathrm{ppb}\end{array}$ \\
\hline & $\begin{array}{l}\text { Teng et al. } \\
(2017)\end{array}$ & China & $\begin{array}{l}\text { Time- } \\
\text { series }\end{array}$ & -/23344(outpatien) & - & ICD-9:477 & $\begin{array}{l}\text { Changchun } \\
\text { Municipal } \\
\text { Environmental } \\
\text { Protection } \\
\text { Monitoring } \\
\text { Center. }\end{array}$ & short-term & $\begin{array}{l}\mathrm{PM}_{2.5}: 66.5 \mu \mathrm{g} / \mathrm{m}^{i} \\
\mathrm{NO}_{2}: 43.6 \mu \mathrm{g} / \mathrm{m}^{3} \\
\text { CO: } 0.93 \mu \mathrm{g} / \mathrm{m}^{3} \\
\text { O3: } 71.1 \mu \mathrm{g} / \mathrm{m}^{3}\end{array}$ \\
\hline Disease & $\begin{array}{l}\text { First } \\
\text { author/ } \\
\text { publication } \\
\text { year }\end{array}$ & Region & $\begin{array}{l}\text { Study } \\
\text { design }\end{array}$ & Sample size/Cases & $\begin{array}{l}\text { Age } \\
\text { (years) }\end{array}$ & ICD & $\begin{array}{l}\text { Data sources } \\
\text { of pollutants }\end{array}$ & Duration & $\begin{array}{l}\text { Mean } \\
\text { Concentration }\end{array}$ \\
\hline & $\begin{array}{l}\text { Hu et al. } \\
\text { (2019) }\end{array}$ & China & $\begin{array}{l}\text { Time- } \\
\text { series }\end{array}$ & $2410392 / 646975$ & $<18$ & ICD-10:J30 & $\begin{array}{l}\text { Shanghai } \\
\text { Environmental } \\
\text { Protection } \\
\text { Agency }\end{array}$ & short-term & $\begin{array}{l}\mathrm{NO}_{2}: 49.1 \mu \mathrm{g} / \mathrm{m}^{3} \\
\mathrm{O}_{3}: 68.5 \mu \mathrm{g} / \mathrm{m}^{3}\end{array}$ \\
\hline & $\begin{array}{l}\text { Chu et al. } \\
\text { (2019) }\end{array}$ & China & $\begin{array}{l}\text { Time- } \\
\text { series }\end{array}$ & $-/ 33063$ & - & $\begin{array}{l}\text { medical } \\
\text { history, } \\
\text { clinical } \\
\text { symptoms, } \\
\text { and the } \\
\text { relevant test }\end{array}$ & $\begin{array}{l}\text { Environmental } \\
\text { Monitoring } \\
\text { Centre }\end{array}$ & short-term & $\begin{array}{l}\mathrm{PM}_{2.5}: 57.3 \mu \mathrm{g} / \mathrm{m}^{\prime} \\
\mathrm{PM}_{10}: 98.9 \mu \mathrm{g} / \mathrm{m}^{3}\end{array}$ \\
\hline
\end{tabular}




\begin{tabular}{|c|c|c|c|c|c|c|c|c|c|}
\hline Disease & $\begin{array}{l}\text { First } \\
\text { author/ } \\
\text { publication } \\
\text { year }\end{array}$ & Region & $\begin{array}{l}\text { Study } \\
\text { design }\end{array}$ & Sample size/Cases & $\begin{array}{l}\text { Age } \\
\text { (years) }\end{array}$ & ICD & $\begin{array}{l}\text { Data sources } \\
\text { of pollutants }\end{array}$ & Duration & $\begin{array}{l}\text { Mean } \\
\text { Concentration }\end{array}$ \\
\hline & $\begin{array}{l}\text { Wang et al. } \\
(2020)\end{array}$ & China & $\begin{array}{l}\text { Time- } \\
\text { series }\end{array}$ & -/14965(outpatient) & - & ICD10:J30 & $\begin{array}{l}\text { China's } \\
\text { National } \\
\text { Urban Air } \\
\text { Quality Real- } \\
\text { time } \\
\text { Publishing } \\
\text { Platform }\end{array}$ & short-term & $\begin{array}{l}\mathrm{PM}_{2.5}: 75.7 \mu \mathrm{g} / \mathrm{m}^{3} \\
\mathrm{PM}_{10}: 132.1 \mu \mathrm{g} / \mathrm{m} \\
\mathrm{SO}_{2}: 33.2 \mu \mathrm{g} / \mathrm{m}^{3} \\
\mathrm{NO}_{2}: 48.4 \mu \mathrm{g} / \mathrm{m}^{3} \\
\mathrm{O}_{3}: 59.4 \mu \mathrm{g} / \mathrm{m}^{3} \\
\mathrm{CO}: 1377 \mu \mathrm{g} / \mathrm{m}^{3}\end{array}$ \\
\hline & $\begin{array}{l}\text { Wang et al. } \\
(2020)\end{array}$ & China & $\begin{array}{l}\text { Time- } \\
\text { series }\end{array}$ & $\begin{array}{l}\text {-/229685(outpatient } \\
\text { visits) }\end{array}$ & - & $\begin{array}{l}\text { ICD-10:J30.4 } \\
01\end{array}$ & $\begin{array}{l}\text { monitoring } \\
\text { station }\end{array}$ & short-term & $\mathrm{PM}_{2.5}: 99.5 \mu \mathrm{g} / \mathrm{m}^{\prime}$ \\
\hline
\end{tabular}

\section{Relationship between air pollution and eczema Effect of long-term air pollution exposure on eczema}

As shown in Table 2 and Figure $\mathrm{S} 1$, long-term exposed to $\mathrm{PM}_{10}$ was found to increase the risk of eczema ( $\mathrm{PM} 10$ : $\mathrm{RR}=1.583,95 \% \mathrm{Cl}$ : 1.328-1.888) with per (10 $\mu \mathrm{g} / \mathrm{m}^{3}$ increase in $\mathrm{PM}_{2.5}$ and $\mathrm{PM}_{10} ; 1 \mathrm{ppb}$ increase in $\mathrm{SO}_{2}, \mathrm{NO}_{2}, \mathrm{CO}$ and $\mathrm{O}_{3}$ ) unit increase in concentration. However, exposed to $\mathrm{PM}_{2.5}, \mathrm{NO}_{2}$ and $\mathrm{SO}_{2}$ was not associated with the risk of eczema $\left(\mathrm{PM}_{2.5}, \mathrm{RR}=1.171,95 \% \mathrm{Cl}: 0.944-1.45 ; \mathrm{NO}_{2}, \mathrm{RR}=1.033,95 \% \mathrm{Cl}: 0.970-1.101 ; \mathrm{SO}_{2}, \mathrm{RR}=1.101,95 \% \mathrm{Cl}\right.$ : 0.897-1.351). The heterogeneity of pooled studies was high in studies that exposed to $\mathrm{PM}_{2.5}\left(\mathrm{PM}_{2.5}: R^{2}=77.97 \%\right)$, whereas low in studies of $\mathrm{PM}_{10}, \mathrm{NO}_{2}$ and $\mathrm{SO}_{2}\left(\mathrm{PM}_{10}: R^{2}=0.00 \%\right.$; $\left.\mathrm{NO}_{2}: P^{2}=0.00 \% ; \mathrm{SO}_{2}: P^{2}=0.00 \%\right)$. There was no publication bias in the combined studies for $\mathrm{PM}_{2.5}$ and $\mathrm{NO}_{2}(P>0.05)$ (Fig. S2a, Fig.S2b). 
Table 2

Pooled estimates of the effect on the risk of diseases

\begin{tabular}{|c|c|c|c|c|c|c|c|}
\hline $\begin{array}{l}\text { Prevalence/incidence } \\
\text { Disease }\end{array}$ & Duration & Pollutants & $\begin{array}{l}\text { Number of } \\
\text { studies }\end{array}$ & $\operatorname{RR}[95 \% \mathrm{Cl}]$ & $R$ & $\begin{array}{l}\text { Pvalue for } \\
\text { heterogeneity }\end{array}$ & $\begin{array}{l}\text { Publication } \\
\text { bias }(p)\end{array}$ \\
\hline \multirow[t]{10}{*}{ Eczema } & Long-term & $\mathrm{PM}_{2.5}$ & 4 & $1.171[0.944,1.453]$ & $77.97 \%$ & 0.0044 & $>0.05$ \\
\hline & & $\mathrm{PM}_{10}$ & 2 & $1.583[1.328,1.888]^{\star}$ & $0.00 \%$ & 0.9654 & - \\
\hline & & $\mathrm{NO}_{2}$ & 6 & $1.033[0.970,1.101]$ & $0.00 \%$ & 0.9050 & $>0.05$ \\
\hline & & $\mathrm{SO}_{2}$ & 1 & $1.101[0.897,1.351]$ & $0.00 \%$ & 1.0000 & - \\
\hline & $\begin{array}{l}\text { Short- } \\
\text { term }\end{array}$ & $\mathrm{PM}_{2.5}$ & 5 & $1.001[0.994,1.007]$ & $72.91 \%$ & 0.0033 & $>0.05$ \\
\hline & & $\mathrm{PM}_{10}$ & 8 & $1.006[1.003,1.008]^{\star}$ & $63.25 \%$ & $<0.0001$ & $>0.05$ \\
\hline & & $\mathrm{NO}_{2}$ & 7 & $1.009[1.008,1.011]^{\star}$ & $10.84 \%$ & 0.2555 & $>0.05$ \\
\hline & & $\mathrm{SO}_{2}$ & 5 & $1.004[0.999,1.009]$ & $12.41 \%$ & 0.4648 & $>0.05$ \\
\hline & & $\mathrm{CO}$ & 1 & $1.000[0.956,1.046]$ & $0.00 \%$ & 1.0000 & - \\
\hline & & $\mathrm{O}_{3}$ & 1 & $0.628[0.342,1.152]$ & $0.00 \%$ & 1.0000 & - \\
\hline \multirow[t]{12}{*}{$A D$} & Long-term & $\mathrm{PM}_{2.5}$ & 4 & $1.153[0.962,1.381]$ & $98.65 \%$ & $<0.0001$ & $>0.05$ \\
\hline & & $\mathrm{PM}_{10}$ & 5 & $1.101[0.947,1.280]$ & $99.29 \%$ & $<0.0001$ & $>0.05$ \\
\hline & & $\mathrm{NO}_{2}$ & 7 & $1.048[0.984,1.116]$ & $97.80 \%$ & $<0.0001$ & $>0.05$ \\
\hline & & $\mathrm{SO}_{2}$ & 4 & $1.223[0.954,1.568]$ & $97.34 \%$ & $<0.0001$ & $>0.05$ \\
\hline & & $\mathrm{CO}$ & 5 & $1.006[0.998,1.013]$ & $73.30 \%$ & 0.0033 & $>0.05$ \\
\hline & & $\mathrm{O}_{3}$ & 5 & $1.010[0.978,1.043]$ & $0.44 \%$ & 0.1920 & $>0.05$ \\
\hline & $\begin{array}{l}\text { Short- } \\
\text { term }\end{array}$ & $\mathrm{PM}_{2.5}$ & 1 & $1.004[1.002,1.007]$ & $0.00 \%$ & 1.0000 & - \\
\hline & & $\mathrm{PM}_{10}$ & 3 & $1.011[0.995,1.028]$ & $98.35 \%$ & 0.0036 & $>0.05$ \\
\hline & & $\mathrm{NO}_{2}$ & 3 & $1.000[0.997,1.004]$ & $0.00 \%$ & 0.8268 & $>0.05$ \\
\hline & & $\mathrm{SO}_{2}$ & 2 & $1.008[1.001,1.015]^{\star}$ & $62.27 \%$ & 0.1035 & - \\
\hline & & $\mathrm{CO}$ & 1 & $1.004[0.999,1.009]$ & $0.00 \%$ & 1.0000 & - \\
\hline & & $\mathrm{O}_{3}$ & 3 & $1.033[0.990,1.078]$ & $82.22 \%$ & 0.0035 & $>0.05$ \\
\hline AR & Long-term & $\mathrm{PM}_{2.5}$ & 7 & $1.058[1.014,1.222]^{\star}$ & $90.81 \%$ & $<0.0001$ & $<0.05$ \\
\hline \multirow{11}{*}{$\begin{array}{l}\text { Prevalence/incidence } \\
\text { Disease }\end{array}$} & Duration & Pollutants & $\begin{array}{l}\text { Number of } \\
\text { studies }\end{array}$ & $\operatorname{RR}[95 \% \mathrm{Cl}]$ & $P^{2}$ & $\begin{array}{l}P \text {-value for } \\
\text { heterogeneity }\end{array}$ & $\begin{array}{l}\text { Publication } \\
\text { bias }(p)\end{array}$ \\
\hline & & $\mathrm{PM}_{10}$ & 8 & $1.004[0.988,1.020]$ & $27.66 \%$ & 0.1230 & $>0.05$ \\
\hline & & $\mathrm{NO}_{2}$ & 11 & $1.003[0.995,1.011]$ & $0.78 \%$ & 0.6720 & $<0.05$ \\
\hline & & $\mathrm{SO}_{2}$ & 8 & $1.014[0.996,1.033]$ & $0.00 \%$ & 0.9395 & $>0.05$ \\
\hline & & $\mathrm{CO}$ & 7 & $1.127[0.893,1.422]$ & $99.68 \%$ & $<0.0001$ & $>0.05$ \\
\hline & & $\mathrm{O}_{3}$ & 11 & $1.004[0.992,1.016]$ & $0.00 \%$ & 0.7592 & $>0.05$ \\
\hline & $\begin{array}{l}\text { Short- } \\
\text { term }\end{array}$ & $\mathrm{PM}_{2.5}$ & 9 & $1.049[0.995,1.107]$ & $99.27 \%$ & $<0.0001$ & $>0.05$ \\
\hline & & $\mathrm{PM}_{10}$ & 11 & $1.028[1.008,1.049]^{*}$ & $98.69 \%$ & $<0.0001$ & $<0.05$ \\
\hline & & $\mathrm{NO}_{2}$ & 9 & $1.018[1.007,1.029]^{\star}$ & $87.91 \%$ & $<0.0001$ & $>0.05$ \\
\hline & & $\mathrm{SO}_{2}$ & 5 & $1.009[1.000,1.018]$ & $83.78 \%$ & $<0.0001$ & $>0.05$ \\
\hline & & $\mathrm{CO}$ & 3 & $1.000[1.000,1.001]$ & $0.00 \%$ & 0.6335 & $>0.05$ \\
\hline
\end{tabular}




\begin{tabular}{|c|c|c|c|c|c|c|c|}
\hline $\begin{array}{l}\text { Prevalence/incidence } \\
\text { Disease }\end{array}$ & Duration & Pollutants & $\begin{array}{l}\text { Number of } \\
\text { studies }\end{array}$ & $\mathrm{RR}[95 \% \mathrm{Cl}]$ & $R$ & $\begin{array}{l}P \text {-value for } \\
\text { heterogeneity }\end{array}$ & $\begin{array}{l}\text { Publication } \\
\text { bias }(p)\end{array}$ \\
\hline & & $\mathrm{O}_{3}$ & 4 & $1.010[0.998,1.022]$ & $68.28 \%$ & 0.0138 & $>0.05$ \\
\hline
\end{tabular}

In subgroup analyses according to age, study types and regions, long-term exposed to $\mathrm{PM}_{2.5}$ and $\mathrm{NO}_{2}$ had no impact on the risk of eczema. Details were shown in Figure 2a. After we conducting subgroup analyses, heterogeneity was still high in $\mathrm{PM}_{2.5}$ subgroups $\left(P^{2}>50 \%\right)$, other undiscovered factors might have impact on the heterogeneity. Furthermore, the studies of $\mathrm{CO}$ and $\mathrm{O}_{3}$ were not enough for subgroup analyses at present.

\section{Effect of short-term air pollution exposure on eczema}

By pooled effect size, we found that short-term exposed to $\mathrm{PM}_{10}$ and $\mathrm{NO}_{2}$ were associated with the risk of eczema $\left(\mathrm{PM} \mathrm{M}_{10}, \mathrm{RR}=1.006,95 \% \mathrm{Cl}\right.$ : $1.003-1.008 ; \mathrm{NO}_{2}$ : $\mathrm{RR}=1.009,95 \% \mathrm{Cl}: 1.008-1.011)$, while exposed to $\mathrm{PM}_{2.5}, \mathrm{SO}_{2}$ did not increase the risk of eczema $\left(\mathrm{PM}_{2.5}, \mathrm{RR}=1.001,95 \% \mathrm{Cl}: 0.994-1.007 ; \mathrm{SO} \mathrm{N}_{2}, \mathrm{RR}=1.004,95 \%\right.$ Cl: 0.999-1.009) (Table 2, Fig. S1). The pooled studies in $\mathrm{PM}_{2.5}$ and $\mathrm{PM}_{10}$ were still high in heterogeneities $\left(P^{2}>50 \%\right)$. There was no publication bias in $\mathrm{PM}_{2.5}$ $\mathrm{PM}_{10}$ and $\mathrm{SO}_{2}$ (Fig. S2 c, Fig. S2d, Fig. S2f). Moreover, the heterogeneity of pooled studies was low in $\mathrm{NO}_{2}$ and there was no publication bias by funnel plot $(P>0.05)$ (Fig. S2e). Studies on pollutants $\mathrm{CO}$ and $\mathrm{O}_{3}$ were not enough for combining analyses at present.

In Figure 2a, the results of analysis on ICD were consistent with no ICD grouping, the combined effects of pollutants $\mathrm{PM}_{10}$ and $\mathrm{NO}_{2}$ were also associated with the risk of eczema $\left(\mathrm{PM}_{10}, \mathrm{RR}=1.005,95 \% \mathrm{Cl}: 1.003-1.008 ; \mathrm{NO}_{2}\right.$ : $\left.\mathrm{RR}=1.009,95 \% \mathrm{Cl}: 1.007-1.011\right)$. In age $<18$ group, $\mathrm{PM}_{2.5}, \mathrm{PM}_{10}$ and $\mathrm{NO}_{2}$ were associated with eczema. Nevertheless, $\mathrm{PM}_{10}$ and $\mathrm{NO}_{2}$ increased the risk of eczema in the all age groups $\left(\mathrm{PM}_{10}, \mathrm{RR}=1.007,95 \% \mathrm{Cl}\right.$ : 1.004-1.009; $\mathrm{NO}$ : $\mathrm{RR}=1.008,95 \% \mathrm{Cl}$ : $1.005-$ 1.010), with low heterogeneity $\left(R_{<}<50 \%\right)$. Combined effect by cross-sectional studies showed no association between short-term exposed to pollutants $\mathrm{PM} \mathrm{M}_{2.5}$ $\mathrm{PM}_{10}, \mathrm{NO}_{2}$ and the risk of eczema. The combination of the time-series studies suggested that these three pollutants increased the risk of eczema ( $\mathrm{PM}_{2.5}$, $\mathrm{RR}=1.003,95 \% \mathrm{Cl}: 1.002-1.004 ; \mathrm{PM}_{10}, \mathrm{RR}=1.007,95 \% \mathrm{Cl}: 1.004-1.009 ; \mathrm{NO}_{2}, \mathrm{RR}=1.008,95 \% \mathrm{Cl}: 1.005-1.010$ ). Furthermore, $\mathrm{PM}_{2.5}, \mathrm{PM}_{10}$, and $\mathrm{NO}_{2}$ exposure were related to eczema in Asia with low heterogeneity $\left(P_{<}<50 \%\right)$.

\section{Relationship between air pollution and $A D$ Effect of long-term air pollution exposure on $A D$}

A total of eleven studies for $A D$ were included in this study. However, significant results were not found in long-term air pollutants exposure ( $P M_{2.5}$, $R R=1.153$, $95 \mathrm{Cl} \%: 0.962,1.381 ; \mathrm{PM}_{10}, \mathrm{RR}=1.101,95 \mathrm{Cl} \%: 0.947,1.280 ; \mathrm{NO}_{2}, \mathrm{RR}=1.048,95 \mathrm{Cl} \%: 0.984,1.116 ; \mathrm{SO}_{2}, \mathrm{RR}=1.223,95 \mathrm{Cl} \%: 0.954,1.568 ; \mathrm{CO}, \mathrm{RR}=1.006,95$ $\mathrm{Cl} \%: 0.998,1.013 ; \mathrm{O}_{3}, \mathrm{RR}=1.010,95 \mathrm{Cl} \%: 0.978,1.043$ ) (Table 2, Fig.S3). The heterogeneities of the combined study were high in $\mathrm{PM}_{2.5}, \mathrm{PM}_{10}, \mathrm{NO}_{2}, \mathrm{SO}_{2}$ and $\mathrm{CO}$ $\left(R^{2}>50 \%\right)$. The funnel plots showed that there were no publication bias $(P>0.05)$ in $\mathrm{PM}_{2.5}, \mathrm{PM}_{10}, \mathrm{NO}_{2}, \mathrm{SO}_{2}, \mathrm{CO}$ and $\mathrm{O}_{3}$ (Figure S4a-4f).

From the Figure 2b, ICD subgroup analyses showed that long-term exposure to six pollutants were not harmful to AD. The results were consistent with the effect of no ICD grouping for AD. However, the results of combined effects through subgroup analyses indicated that $\mathrm{PM}_{10}$ and $\mathrm{CO}$ were harmful to the occurrence of $A D\left(P M_{10}, R R=1.049,95 \% \mathrm{Cl}: 1.010-1.089 ; \mathrm{CO}: \mathrm{RR}=1.009,95 \% \mathrm{Cl}\right.$ : 1.006-1.012) with low heterogeneity $\left(R^{2}<50 \%\right)$ in age<18, while $\mathrm{PM} 2.5, \mathrm{NO}_{2}, \mathrm{SO}{ }_{2}$ and $\mathrm{O}_{3}$ were irrelevant. Subgroup analyses of study type was found a significant association between $\mathrm{AD}$ and $\mathrm{SO}_{2}\left(\mathrm{RR}=1.622,95 \% \mathrm{Cl}: 1.556-1.690, R^{2}=0.00 \%\right)$ in cohort studies. No positive results were discovered for the combination of cross-sectional studies effects. In addition, the subgroup analyses showed no association between $A D$ and the pollutants in Asian.

\section{Effect of short-term air pollution exposure on $A D$}

As we can see from Table 2 and Figure $\mathrm{S} 3$, short-term exposure to $\mathrm{SO}_{2}$ increased the risk of $\mathrm{AD}$ by 1.008 per unit of concentration (RR: 1.008 , $95 \%$ Cl: 1.001 1.015, $P=62.27 \%) . \mathrm{PM}_{10}, \mathrm{NO}_{2}$ and $\mathrm{O}_{3}$ had no adverse effect on $\mathrm{AD}$ with per unit increase in concentration (PM $10, \mathrm{RR}: 1.011,95 \%$ Cl: 0.995-1.028; $\mathrm{NO}_{2}, \mathrm{RR}$ : $1.000,95 \% \mathrm{Cl}: 0.997-1.004 ; \mathrm{O}_{3}, \mathrm{RR}: 1.033,95 \% \mathrm{Cl}: 0.990-1.078$ ). Since only one research article studied on $\mathrm{PM} \mathrm{M}_{2.5}$, $\mathrm{CO}$ and $\mathrm{AD}$, they cannot be combined for a further analysis.

In Figure $2 \mathrm{~b}$, the sensitivity analyses of ICD groups showed that $\mathrm{PM}_{10}$ (RR: $\left.1.003,95 \% \mathrm{Cl}: 1.002-1.005, P=0.00 \%\right), \mathrm{SO}_{2}(\mathrm{RR}: 1.008,95 \% \mathrm{Cl}: 1.001-1.015$, $P^{2}=0.00 \%$ ) and $\mathrm{O}_{3}$ (RR: $1.012,95 \% \mathrm{Cl}: 1.007-1.017, P^{2}=0.00 \%$ ) were correlation with $\mathrm{AD}$ in short time. Analyses of $\mathrm{SO}_{2}$ and $\mathrm{O}_{3}$ by $\mathrm{ICD}$ showed that increased the risk of $A D$ with per unit increase in concentration $\left(\mathrm{SO}_{2}\right.$ : RR: $1.008,95 \% \mathrm{Cl}: 1.001-1.015 ; \mathrm{O}_{3}, \mathrm{RR}$ : $1.012,95 \%$ Cl: 1.007-1.017). In different age groups, $\mathrm{PM} \mathrm{M}_{10}$ increased the risk of $A D$ in the general population. Furthermore, $\mathrm{NO}_{2}$ and $\mathrm{O}_{3}$ did not increase $A D$ 's risk for the population aged<18. What's more, air pollutants were not associated with $A D$ risk in subgroup analyses of study types and regions.

\section{Relationship between air pollution and AR Effect of long-term air pollution exposure on AR}

The results showed that only long-term exposure to $\mathrm{PM}_{2.5}$ had a harmful effect to the occurrence of AR (RR=1.058, 95\% Cl: 1.014-1.222; Table 2, Fig.S5). But the heterogeneity of the combined articles was high $\left(R^{2}=90.81 \%\right)$, and the included articles had publication bias $(P<0.05)\left(\mathrm{Fig}^{\mathrm{S}} \mathrm{S} 6 \mathrm{a}\right) . \mathrm{PM}_{10}, \mathrm{NO}_{2}, \mathrm{SO}_{2}, \mathrm{CO}_{3} \mathrm{O}_{3}$

Page 13/19 
had no effect on the risk of $\mathrm{AR}$, and the details were shown in supplementary material Figure $\mathrm{S} 5$. In addition, the articles studying $\mathrm{PM}_{10}, \mathrm{SO}_{2}, \mathrm{CO}$ and $\mathrm{O}_{3}$ had no publication bias $(P>0.05)$ (Fig. S6b, S6d-S6f), while that studying $\mathrm{NO}_{2}$ had publication bias $(P<0.05)($ Fig. S6c).

According to subgroup analyses in Figure 2c, combined analyses of articles classified by ICD showed no association between $\mathrm{PM}_{2.5}, \mathrm{O}_{3}$ and $A R\left(P M_{2.5}\right.$, $\mathrm{RR}=1.145,95 \% \mathrm{Cl}: 0.885-1.480 ; \mathrm{O}_{3}, \mathrm{RR}=1.040,95 \% \mathrm{Cl}: 0.949-1.140$. Additionally, only $\mathrm{PM}_{2.5}$ increased the risk of $A R$ in the age $<18 \mathrm{group}\left(\mathrm{PM} \mathrm{M}_{2.5}\right.$ : $\mathrm{RR}=1.133$, $95 \% \mathrm{Cl}: 1.017-1.262)$. The pooled $\mathrm{RR}$ of $\mathrm{PM}_{10}$ was significant in cross-sectional studies $\left(\mathrm{PM}_{10}: \mathrm{RR}=1.021,95 \% \mathrm{Cl}: 1.003-1.038, P^{2}=0.00 \%\right)$. In addition, $\mathrm{PM} 2.5$ was also found to be strengthened the risk of $A R$ in Asia $\left(P_{2.5}: R R=1.222,95 \%\right.$ Cl: 1.098-1.361), while other pollutants did not have a positive relationship with AR for Europe.

\section{Effect of short-term air pollution exposure on AR}

As shown in Table 2 and Figure S5, short-term exposure to $\mathrm{PM}_{10}$ and $\mathrm{NO}_{2}$ were associated with the risk of $A R\left(P M_{10}: R R=1.028,95 \%\right.$ Cl: $1.008-1.049 ; \mathrm{NO}_{2}$ : $\mathrm{RR}=1.018,95 \% \mathrm{Cl}: 1.007-1.029)$. However, $\mathrm{PM}_{2.5}, \mathrm{SO}_{2}, \mathrm{CO}$ and $\mathrm{O}_{3}$ were not associated with the risk of $\mathrm{AR}\left(\mathrm{PM}_{2.5}: \mathrm{RR}=1.049,95 \% \mathrm{Cl}: 0.995-1.107 ; \mathrm{SO}\right.$ : $\left.\mathrm{RR}=1.009,95 \% \mathrm{Cl}: 1.000-1.018 ; \mathrm{CO}: \mathrm{RR}=1.000,95 \% \mathrm{Cl}: 1.000-1.001 ; \mathrm{O}_{3}: \mathrm{RR}=1.010,95 \% \mathrm{Cl}: 0.998-1.022\right)$. Except for CO, the heterogeneity was higher $\left(\mathrm{PM} 2.5:{ }^{2}=\right.$ $\left.99.27 \%, \mathrm{PM}_{10}: P^{2}=98.69 \%, \mathrm{NO}_{2}: P^{2}=87.91 \%, \mathrm{SO}_{2}: P^{2}=83.78 \%, \mathrm{O}_{3}: P^{2}=68.28 \%\right)$. There was no publication bias for the pollutant studies $(P>0.05)$ besides $\mathrm{PM}_{10}$ (Fig. S6g-S6i).

As shown in Figure 2c, the subgroups of ICD, $\mathrm{PM}_{10}, \mathrm{NO}_{2}$ and $\mathrm{SO}_{2}$ were harmful factors for $A R\left(P M_{10}, \mathrm{RR}=1.030,95 \% \mathrm{Cl}: 1.004-1.056 ; \mathrm{NO} \mathrm{N}_{2}, \mathrm{RR}=1.022,95 \% \mathrm{Cl}\right.$ : 1.009-1.035; $\left.\mathrm{SO}_{2}, \mathrm{RR}=1.016,95 \% \mathrm{Cl}: 1.003-1.030\right)$, but the heterogeneity of included articles were high $\left(P^{2>} 50 \%\right)$. Through the ICD subgroup, we found that $\mathrm{SO}{ }_{2}$ increases AR's risk. In analysis of age, $\mathrm{PM}_{10}$ was harmful to age $<18$. For all age group, $\mathrm{PM}_{2.5}, \mathrm{PM}_{10}, \mathrm{NO}_{2}$ and $\mathrm{SO}_{2}$ increased the risk of $A R\left(P M_{2.5}, \mathrm{RR}=1.005\right.$, 95\% Cl: 1.003-1.007; $\mathrm{PM}_{10}, \mathrm{RR}=1.012,95 \% \mathrm{Cl}: 1.003-1.021 ; \mathrm{NO}_{2}, \mathrm{RR}=1.016,95 \% \mathrm{Cl}: 1.008-1.024 ; \mathrm{SO}_{2}, \mathrm{RR}=1.016,95 \% \mathrm{Cl}: 1.003-1.030$ ) with low heterogeneity $\left(R^{2}<50 \%\right)$. In addition, $\mathrm{PM}_{10}$ and $\mathrm{NO}_{2}$ were potential risk factors for $\mathrm{AR}\left(\mathrm{PM}_{10}, \mathrm{RR}: 1.022,95 \% \mathrm{Cl}: 1.003-1.041 ; \mathrm{NO}_{2}, \mathrm{RR}: 1.018,95 \%\right.$ Cl: $\left.1.007-1.028\right)$ in timeseries studies with high heterogeneity $\left(\mathrm{PM}_{10}: R^{2}=98.88 \%, \mathrm{NO}_{2}: R^{2}=91.23 \%\right)$. In subgroup analyses on region, the impacts of $\mathrm{PM}_{10}$ and $\mathrm{NO}_{2}$ for $\mathrm{AR}^{2}$ were detrimental in Asia ( $\mathrm{PM}_{10}$, RR: 1.023, 95\% Cl: 1.005-1.041; $\mathrm{NO}_{2}$, RR: 1.017, 95\% Cl: 1.004-1.029). But the heterogeneity of both were still high ( $\mathrm{PM} \mathrm{M}_{10}$ : $P^{2}=98.34 \%$; $\left.\mathrm{NO}_{2}: R=90.31 \%\right)$, which may be affected other factors.

\section{Discussion}

This meta-analysis has several main findings. (1) Long-term exposure to $\mathrm{PM}_{10}$ increased the risk of eczema. (2) Long-term exposure PM 2.5 was associated with AR. (3) Short-term exposure to $\mathrm{PM}_{10}$ and $\mathrm{NO}_{2}$ increased the risk for eczema and AR. (4) Short-term exposure to $\mathrm{SO}_{2}$ was associated with the development of $A D$. In addition, significant results were found in subgroup analyses. (1) By ICD classification analyses, long-term exposure to PM 10 increased the risk of eczema, $A D$ and $A R$. (2) In age<18 years old group, long-term exposure to $\mathrm{PM}_{10}, \mathrm{PM}_{2.5}$ increased the risk of $A D$ and $A R$ respectively. For all age group, shortterm exposure to $\mathrm{PM}_{10}$ and $\mathrm{NO}_{2}$ were associated with eczema and $A R$. (3) $\mathrm{SO}_{2}$ has been found to increase the risk of $A D$ in cohort studies and $P M_{10}$ and $\mathrm{NO}_{2}$ were correlated with eczema and $A R$ in time series studies types. (4) Long-term exposure to $\mathrm{PM}_{2.5}$ increased the risk of Asia's AR patients and short-term exposure to $\mathrm{PM}_{10}$ and $\mathrm{NO}_{2}$ were associated with the development of eczema and $\mathrm{AR}$.

To our knowledge, this is up-to-date meta-analysis and review of current evidence between air pollutants and lgE mediated allergic disease. There were metaanalyses on IgE mediated allergic diseases. About eczema, one meta-analysis has been studied by Fuertes et al. found no association between $\mathrm{PM}_{2.5}, \mathrm{NO}_{2}$ and children's eczema (Fuertes et al., 2020). Our results also suggested that long-term exposure to $\mathrm{PM}_{2.5}$ and $\mathrm{NO}_{2}$ were not associated with eczema in children. In addition, our results complemented that long-term exposure to $\mathrm{PM}_{10}$ and $\mathrm{NO}_{2}$ increased the risk of eczema in all age group. Subgroup analyses of study design and regions were also performed and the results were innovative. In 2014, a meta-analysis on allergy and sensitization of traffic-related air pollution showed that two of birth cohorts reported adverse effects of $\mathrm{NO}_{2}$ on the prevalence of eczema (Bowatte et al., 2015). Our meta-analysis updated the risk assessment of $\mathrm{NO}_{2}$ on children's eczema. Besides $\mathrm{AD}$ has not been studied for a meta-analysis. AR, Zou et al. included thirteen studies for meta-analysis and found that when exposed to $\mathrm{NO}_{2}, \mathrm{SO}_{2}, \mathrm{PM}_{10}$ and $\mathrm{PM}_{2.5}$, the prevalence of childhood AR might increase (Zou et al., 2018). In a meta-analysis of particulate matter (PM) and the prevalence of AR in children published in 2021, Lin et al. had reported the relation between $\mathrm{PM}_{2.5}, \mathrm{PM}_{10}$ and childhood $A R$. In our study, we considered the effects of long-term and short-term exposure to pollutants, the results shown that long-term exposure to $\mathrm{PM}_{2.5}$ increased the risk of children's AR and short-term exposure to $\mathrm{PM}_{10}$ increased the risk of children's AR. Moreover, we performed subgroup analyses of ICD, study types, and age.

Air pollution has become a public health problem, and was the most important cause of premature death in 2015 (Combes and Franchineau, 2019 ). Air pollution is widely considered as a detrimental factor for the lungs, respiratory tract and most organ systems. At the same time, air pollution affects the human immune system, that might be related to allergic sensitization and the occurrence of autoimmune diseases (Schraufnagel et al., 2019). Li et al. found that $\mathrm{PM}_{2.5}$ could cause dermatitis by promoting the expression of thymic stromal lymphopoietin in keratinocytes (Li et al., 2020). In a BALB/c mouse model of $\mathrm{PM}_{2.5}$ exposure mechanism study, nucleotide-binding oligomerization domain-containing protein 1(Nod 1) and nuclear factor (NF)- $\mathrm{KB}$ signaling pathway would be activated after exposing to $\mathrm{PM}_{2.5}$, then cause pro-inflammatory reactions (Manzo et al., 2012). PM 10 might induce NF-kB activation in human airway epithelial cells, and this process is mediated by oxidation mechanisms (Salvi, 2001). Exposure to air pollution increases the increase of eosinophils (EOS) in the nasal mucosa. A mechanism study in female mouse reported that $\mathrm{NO}_{2}$ was an endogenous mediator of inflammation. After exposure, mixed Th2/Th17 adaptive immune response and neutrophils would appear. EOS and neutrophils were recruited to the airway, causing inflammation (Martin et al., 2013) (Fig. 3). 
Air pollutants could be absorbed into subcutaneous tissues by hair follicles and sweat/sebaceous glands of the skin. When the defense capability is exceeded, the function of the skin will be impaired and may lead to the occurrence of skin diseases (Puri et al., 2017). A study conducted by Park et al. found that $\mathrm{PM}_{10}$ could increase the expression of NF-KB, IL-1, IL-6, IL-8 and IL-33 genes in dermal fibroblasts, thereby transcribing pro-inflammatory genes and

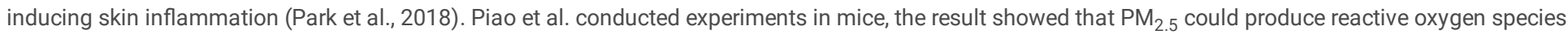
ROS and participates in oxidative stress, this can cause DNA damage, lipid peroxidation and protein carbonylation (Piao et al., 2018). When normal human epidermal keratinocytes are exposed to PM, the aromatic hydrocarbon receptor (AHR) is activated. AHR pathway and ROS induce pro-inflammatory factors. The activation of AHR is related to human atopic dermatitis (Araviiskaia et al., 2019). In short, PM exposure increased pro-inflammatory mediators and the expression of AHR, then increased ROS. Finally, skin damage is mainly caused by PM-induced oxidative stress. Moreover, it has been reported that $\mathrm{NO}_{2}$ might damage the barrier function of the skin (Eberlein-König et al., 1998). The above is related to the pathology of dermatitis (Fig. 3). At present, the mechanism between air pollution and IgE immune-mediated diseases is not yet clear. and it might also be related to human susceptibility (Kimata, 2004; Schmitz et al., 2012). Therefore, more mechanisms in this area need to be studied. Only in this way can we prevent the occurrence of the disease from the root cause.

The advantages of this analysis were including update studies of short-term and long-term exposure to air pollutants. There were six pollutants and a larger sample size to analyze the pooled effects and the results were more comprehensive. Then, based on the widely accepted NOS and OHAT inclusion of the study tools, the quality and risk of deviations were carefully assessed. All included studies had high quality. At last, all effect values were standardized for further analyses. However, the study limitations should be considered. The existing articles on air pollutants are not sufficient for analysis, more studies related to the effects of air pollution on IgE mediated allergic disease are still needed.

In conclusion, this comprehensive meta-analysis found that particulate pollutants $\left(\mathrm{PM}_{2.5}\right.$ and $\left.\mathrm{PM}_{10}\right), \mathrm{NO}_{2}$, and $\mathrm{SO}_{2}$ were potentially harmful to IgE-mediated immune diseases. Air pollutants may be emerging risk factors for allergic diseases. Therefore, the underlying factors shouldn't be ignored. More attention should be paid to vulnerable population, especially for children. Moreover, strengthen protective measures should be conducted to reduce the incidence of allergic diseases, alleviate people from diseases and save medical resources.

\section{Declarations}

Ethical Approval[Not applicable.

Consent to Participate: Not applicable (This study does not contain any individual person's data in any form).

Consent to Publish: The authors declare that they agree with the publication of this paper in this journal.

\section{Author Contributions}

Hua Wang: performed the data analysis, writing-original draft. Xian-Bao Li: performed the data analysis. Xiu-Jie Chu: investigation, data curation. Nv-Wei Cao: formal analysis, helped revise the manuscript. Hong Wu: investigation. Rong-Gui Huang: investigation. Bao-Zhu Li (Ibz88730@163.com) and Dong-Qing Ye (anhuiydq@126.com): conceptualization, project administration, funding acquisition, writing-original draft.

Funding: This study was supported by National Natural Science Foundation of China [81803310]; Undergraduate Innovation and Entrepreneurship Training Program in Anhui Province [S201910366064]; Emergency research project of novel coronavirus infection of Anhui Medical University [YJGG202003]; and the Grants for Scientific Research of BSKY [XJ201619] from Anhui Medical University; Research Fund of Anhui Institute of translational medicine [2021zhyx-C21].

Competing Interests: There were no conflict interests in authors.

Availability of data and materials: Not applicable.

\section{Acknowledgement}

We thank the participants for joining our study and reviewers for their valuable suggestion.

\section{References}

1. Aguilera I, Pedersen M, Garcia-Esteban R et al (2013) Early-life exposure to outdoor air pollution and respiratory health, ear infections, and eczema in infants from the INMA study. Environ Health Perspect 121:387-392

2. Aini NR, Mohd Noor N, Md Daud MK, Wise SK, Abdullah B (2021) Efficacy and safety of intralymphatic immunotherapy in allergic rhinitis: A systematic review and meta-analysis.Clinical and translational allergy $11, \mathrm{e} 12055$

3. Anderson HR, Ruggles R, Pandey KD et al (2010) Ambient particulate pollution and the world-wide prevalence of asthma, rhinoconjunctivitis and eczema in children: Phase One of the International Study of Asthma and Allergies in Childhood (ISAAC). Occupational and environmental medicine 67, 293-300

4. Araviiskaia E, Berardesca E, Bieber T et al (2019) The impact of airborne pollution on skin. Journal of the European Academy of Dermatology and Venereology: JEADV 33:1496-1505

5. Archer CB (2021) Atopic dermatitis. Medicine 49:370-373

6. Arnedo-Pena A, García-Marcos L, Carvajal Urueña I et al (2009) Air pollution and recent symptoms of asthma, allergic rhinitis, and atopic eczema in schoolchildren aged between 6 and 7 years. Arch Bronconeumol 45:224-229

7. Baek JO, Cho J, Roh JY (2021) Associations between ambient air pollution and medical care visits for atopic dermatitis. Environ Res 195:110153 
8. Bai W, Li Y, Niu Y et al (2020) Association between ambient air pollution and pregnancy complications: A systematic review and meta-analysis of cohort studies. Environ Res 185:109471

9. Belugina IN, Yagovdik NZ, Belugina OS, Belugin SN (2018) Outdoor environment, ozone, radionuclide-associated aerosols and incidences of infantile eczema in Minsk, Belarus. Journal of the European Academy of Dermatology and Venereology: JEADV 32:1977-1985

10. Bowatte G, Lodge C, Lowe AJ et al (2015) The influence of childhood traffic-related air pollution exposure on asthma, allergy and sensitization: a systematic review and a meta-analysis of birth cohort studies. Allergy 70:245-256

11. Brauer M, Hoek G, Smit HA et al (2007) Air pollution and development of asthma, allergy and infections in a birth cohort. Eur Respir J 29:879-888

12. Burte E et al (2018) Association between air pollution and rhinitis incidence in two European cohorts. Environ Int 115:257-266

13. Cai J, Li B, Yu W et al (2020) Associations of household dampness with asthma, allergies, and airway diseases among preschoolers in two crosssectional studies in Chongqing, China: Repeated surveys in 2010 and 2019. Environ Int 140:105752

14. Chen CC, Chiu HF, Yang CY (2016a) Air pollution exposure and daily clinical visits for allergic rhinitis in a subtropical city: Taipei, Taiwan. J Toxicol Environ Health A 79:494-501

15. Chen F et al (2018) The effects of PM(2.5) on asthmatic and allergic diseases or symptoms in preschool children of six Chinese cities, based on China, Children, Homes and Health (CCHH) project. Environmental pollution (Barking, Essex: 1987) 232, 329-337

16. Chen J, Peng L, He S, Li Y, Mu Z (2016b) Association between environmental factors and hospital visits among allergic patients: A retrospective study. Asian Pac J Allergy Immunol 34:21-29

17. Chen JP, Chen GC, Wang XP, Qin L, Bai Y (2017) Dietary Fiber and Metabolic Syndrome: A Meta-Analysis and Review of Related Mechanisms. Nutrients 10

18. Chevalier C, Stojanović O, Colin DJ et al (2015) Gut Microbiota Orchestrates Energy Homeostasis during Cold. Cell 163:1360-1374

19. Chu H, Xin J, Yuan Q, Wang M, Cheng L, Zhang Z, Lu M (2019) The effects of particulate matters on allergic rhinitis in Nanjing, China. Environ Sci Pollut Res Int 26:11452-11457

20. Chung HY, Hsieh CJ, Tseng CC, Yiin LM (2016) Association between the First Occurrence of Allergic Rhinitis in Preschool Children and Air Pollution in Taiwan.Int J Environ Res Public Health13

21. Combes A, Franchineau G (2019) Fine particle environmental pollution and cardiovascular diseases. Metabolism: clinical and experimental 100 s, 153944

22. de Marco R et al (2002) The impact of climate and traffic-related NO2 on the prevalence of asthma and allergic rhinitis in Italy. Clinical and experimental allergy: journal of the British Society for Allergy and Clinical Immunology 32:1405-1412

23. Deng S, Huang D, Wang W, Yan H, Li S, Xiang H (2019) Associations of gestational and the first year of life exposure to ambient air pollution with childhood eczema in Hubei, China. Environ Sci Pollut Res Int 26:23842-23849

24. Diaz-Sanchez D, Tsien A, Fleming J, Saxon A (1997) Combined diesel exhaust particulate and ragweed allergen challenge markedly enhances human in vivo nasal ragweed-specific IgE and skews cytokine production to a T helper cell 2-type pattern. Journal of immunology (Baltimore, Md: 1950) 158, 240613

25. Dong YM, Liao LY, Li L, Yi F, Meng H, He YF, Guo MM (2019) Skin inflammation induced by ambient particulate matter in China. Sci Total Environ 682:364-373

26. Eberlein-König B, Przybilla B, Kühnl P et al (1998) Influence of airborne nitrogen dioxide or formaldehyde on parameters of skin function and cellular activation in patients with atopic eczema and control subjects. J Allergy Clin Immunol 101:141-143

27. Edwards L, Wilkinson P, Rutter G, Milojevic A (2022) Health effects in people relocating between environments of differing ambient air pollution concentrations: A literature review. Environ Pollut 292:118314

28. Fan SJ, Heinrich J, Bloom MS et al (2020) Ambient air pollution and depression: A systematic review with meta-analysis up to 2019. Sci Total Environ 701:134721

29. Fuertes E et al (2013a) : Childhood allergic rhinitis, traffic-related air pollution, and variability in the GSTP1, TNF, TLR2, and TLR4 genes: results from the TAG Study. The Journal of allergy and clinical immunology 132, 342-52.e2

30. Fuertes E, Standl M, Cyrys J et al (2013b) A longitudinal analysis of associations between traffic-related air pollution with asthma, allergies and sensitization in the GINIplus and LISAplus birth cohorts.PeerJ1, e193

31. Fuertes E et al (2020) Associations between air pollution and pediatric eczema, rhinoconjunctivitis and asthma: A meta-analysis of European birth cohorts. Environ Int 136:105474

32. Gehring U, Wijga AH, Brauer M et al (2010) Traffic-related air pollution and the development of asthma and allergies during the first 8 years of life. Am $\mathrm{J}$ Respir Crit Care Med 181:596-603

33. Guo Q, Liang F, Tian L, Schikowski T, Liu W, Pan X (2019) Ambient air pollution and the hospital outpatient visits for eczema and dermatitis in Beijing: a time-stratified case-crossover analysis. Environmental science Processes \& impacts 21:163-173

34. Hao S, Yuan F, Pang P, Yang B, Jiang X, Yan A (2021) Early childhood traffic-related air pollution and risk of allergic rhinitis at 2-4 years of age modification by family stress and male gender: a case-control study in Shenyang, China. Environ Health Prev Med 26:48

35. Hu J, Chen J, Ye L, Cai Z, Sun J, Ji K (2018) Anti-IgE therapy for IgE-mediated allergic diseases: from neutralizing IgE antibodies to eliminating IgE(+) B cells. Clinical and translational allergy 8:27

36. Hu Y, Xu Z, Jiang F et al (2020) Relative impact of meteorological factors and air pollutants on childhood allergic diseases in Shanghai, China. Sci Total Environ 706:135975 
37. Huang J, Choo YJ, Smith HE, Apfelbacher C (2021) Quality of life in atopic dermatitis in Asian countries: a systematic review. Archives of dermatological research

38. Huang Q, Ren Y, Liu Y et al (2019) Associations of gestational and early life exposure to air pollution with childhood allergic rhinitis. Atmos Environ 200:190-196

39. Hüls A et al (2018) Atopic dermatitis: Interaction between genetic variants of GSTP1, TNF, TLR2, and TLR4 and air pollution in early life. Pediatric allergy and immunology: official publication of the European Society of Pediatric Allergy and Immunology 29:596-605

40. Hüls A, Abramson MJ, Sugiri D, Fuks K, Krämer U, Krutmann J, Schikowski T (2019) Nonatopic eczema in elderly women: Effect of air pollution and genes. J Allergy Clin Immunol 143:378-385e9

41. Hwang BF, Jaakkola JJ, Lee YL, Lin YC, Guo YL (2006) Relation between air pollution and allergic rhinitis in Taiwanese schoolchildren. Respir Res 7:23

42. Ibrahim MF, Hod R, Nawi AM, Sahani M (2021) Association between ambient air pollution and childhood respiratory diseases in low- and middle-income Asian countries: A systematic review. Atmos Environ 256:118422

43. Jiang W, Lu C, Miao Y, Xiang Y, Chen L, Deng Q (2018) Outdoor particulate air pollution and indoor renovation associated with childhood pneumonia in China. Atmos Environ 174:76-81

44. Jo EJ, Lee WS, Jo HY et al (2017) Effects of particulate matter on respiratory disease and the impact of meteorological factors in Busan. Korea Respiratory medicine 124:79-87

45. Karagün E, Yıldız P, Cangür Ş (2021) Effects of climate and air pollution factors on outpatient visits for eczema: a time series analysis. Arch Dermatol Res 313:49-55

46. Kathuria P, Silverberg $\mathrm{JI}$ (2016) Association of pollution and climate with atopic eczema in US children. Pediatric allergy and immunology: official publication of the European Society of Pediatric Allergy and Immunology 27:478-485

47. Kim BJ, Kwon JW, Seo JH et al (2011) Association of ozone exposure with asthma, allergic rhinitis, and allergic sensitization. Annals of allergy, asthma \& immunology: official publication of the American College of Allergy, Asthma, \& Immunology 107, 214-9.e1

48. Kim J, Han Y, Seo SC et al (2016) Association of carbon monoxide levels with allergic diseases in children. Allergy and asthma proceedings 37 , e1-7

49. Kim SH, Lee J, Oh I et al (2021) Allergic rhinitis is associated with atmospheric SO2: Follow-up study of children from elementary schools in Ulsan, Korea.PLoS One16, e0248624

50. Kim YM, Kim J, Han Y, Jeon BH, Cheong HK, Ahn K (2017) : Short-term effects of weather and air pollution on atopic dermatitis symptoms in children: A panel study in Korea.PloS one12, e0175229

51. Kimata $\mathrm{H}$ (2004) Exposure to road traffic enhances allergic skin wheal responses and increases plasma neuropeptides and neurotrophins in patients with atopic eczema/dermatitis syndrome. Int J Hyg Environ Health 207:45-49

52. Krämer U, Sugiri D, Ranft U et al (2009) Eczema, respiratory allergies, and traffic-related air pollution in birth cohorts from small-town areas. J Dermatol Sci 56:99-105

53. Lee JT, Cho YS, Son JY (2010) Relationship between ambient ozone concentrations and daily hospital admissions for childhood asthma/atopic dermatitis in two cities of Korea during 2004-2005. Int J Environ Health Res 20:1-11

54. Li A, Fan L, Xie L, Ren Y, Li L (2018) Associations between air pollution, climate factors and outpatient visits for eczema in West China Hospital, Chengdu, south-western China: a time series analysis. Journal of the European Academy of Dermatology and Venereology: JEADV 32:486-494

55. Li F, Dong Y, Ni C, Kan H, Yan S (2020) Fine Particulate Matter (PM2.5) is a Risk Factor for Dermatitis by Promoting the Expression of Thymic Stromal Lymphopoietin (TSLP) in Keratinocytes. Indian journal of dermatology 65:92-96

56. Li Q, Yang Y, Chen R, Kan H, Song W, Tan J, Xu F, Xu J (2016) Ambient Air Pollution, Meteorological Factors and Outpatient Visits for Eczema in Shanghai, China: A Time-Series Analysis.International journal of environmental research and public health13

57. Lin L, Li T, Sun M, Liang Q, Ma Y, Wang F, Duan J, Sun Z (2021a) Effect of particulate matter exposure on the prevalence of allergic rhinitis in children: A systematic review and meta-analysis. Chemosphere 268:128841

58. Lin YT, Shih H, Jung CR, Wang CM, Chang YC, Hsieh CY, Hwang BF (2021b) Effect of exposure to fine particulate matter during pregnancy and infancy on paediatric allergic rhinitis. Thorax 76:568-574

59. Liu K et al (2020) Benefits of influenza vaccination on the associations between ambient air pollution and allergic respiratory diseases in children and adolescents: New insights from the Seven Northeastern Cities study in China. Environmental pollution (Barking, Essex: 1987) 256, 113434

60. Liu W, Cai J, Huang C, Hu Y et al (2016) Associations of gestational and early life exposures to ambient air pollution with childhood atopic eczema in Shanghai, China. The Science of the total environment 572,34-42

61. Lopez DJ, Lodge CJ, Bui DS et al (2021) Association between ambient air pollution and development and persistence of atopic and non-atopic eczema in a cohort of adults. Allergy 76:2524-2534

62. Lu C, Deng QH, Ou CY, Liu WW, Sundell J (2013) Effects of ambient air pollution on allergic rhinitis among preschool children in Changsha, China. Chin Sci Bull 58:4252-4258

63. Manzo ND, LaGier AJ, Slade R, Ledbetter AD, Richards JH, Dye JA (2012) Nitric oxide and superoxide mediate diesel particle effects in cytokine-treated mice and murine lung epithelial cells-implications for susceptibility to traffic-related air pollution. Part Fibre Toxicol 9:43

64. Martin RA, Ather JL, Daggett R, Hoyt L, Alcorn JF, Suratt BT, Weiss DJ, Lundblad LK, Poynter ME (2013) The endogenous Th17 response in NO2-promoted allergic airway disease is dispensable for airway hyperresponsiveness and distinct from Th17 adoptive transfer.PloS one8, e74730 
65. Marx T, Bernard N, Kepka S, Gérazime A, Mauny F, Desmettre T (2021) Pneumothorax and the environment: A systematic review of the impact of air pollution and meteorology, and a meta-analysis on meteorology factors. Environmental pollution (Barking, Essex: 1987) 283, 117089

66. Min KD, Yi SJ, Kim HC, Leem JH, Kwon HJ, Hong S, Kim KS, Kim SY (2020) Association between exposure to traffic-related air pollution and pediatric allergic diseases based on modeled air pollution concentrations and traffic measures in Seoul, Korea: a comparative analysis. Environmental health: a global access science source 19, 6

67. Morgan RL, Whaley P, Thayer KA, Schünemann HJ (2018) Identifying the PECO: A framework for formulating good questions to explore the association of environmental and other exposures with health outcomes. Environ Int 121:1027-1031

68. Murrison LB, Brandt EB, Myers JB, Hershey GKK (2019) Environmental exposures and mechanisms in allergy and asthma development. J Clin Investig 129:1504-1515

69. Mustafic H, Jabre P, Caussin C et al (2012) Main air pollutants and myocardial infarction: a systematic review and meta-analysis. JAMA 307:713-721

70. Neuhaus-Steinmetz U, Uffhausen F, Herz U, Renz H (2000) Priming of allergic immune responses by repeated ozone exposure in mice. Am J Respir Cell Mol Biol 23:228-233

71. Ning J, Zhang Y, Hu H, Hu W, Li L, Pang Y, Ma S, Niu Y, Zhang R (2021) Association between ambient particulate matter exposure and metabolic syndrome risk: A systematic review and meta-analysis. Sci Total Environ 782:146855

72. Ntarladima AM, Vaartjes I, Grobbee DE et al (2019) Relations between air pollution and vascular development in 5-year old children: a cross-sectional study in the Netherlands. Environmental health: a global access science source 18:50

73. Park SK, Kim JS, Seo HM (2021) Exposure to air pollution and incidence of atopic dermatitis in the general population: A national population-based retrospective cohort study. Journal of the American Academy of Dermatology

74. Park SY, Byun EJ, Lee JD, Kim S, Kim HS (2018) : Air Pollution, Autophagy, and Skin Aging: Impact of Particulate Matter (PM(10)) on Human Dermal Fibroblasts. International journal of molecular sciences 19

75. Piao MJ, Ahn MJ, Kang KA et al (2018) Particulate matter 2.5 damages skin cells by inducing oxidative stress, subcellular organelle dysfunction, and apoptosis. Arch Toxicol 92:2077-2091

76. Puri P, Nandar SK, Kathuria S, Ramesh V (2017) Effects of air pollution on the skin: A review. Indian J Dermatol Venereol Leprol 83:415-423

77. Renz H, Conrad M, Brand S, Teich R, Garn H, Pfefferle PI (2011) Allergic diseases, gene-environment interactions. Allergy 66(Suppl 95):10-12

78. Rożalski M, Rudnicka L, Samochocki Z (2016) Atopic and Non-atopic Eczema. Acta dermatovenerologica Croatica: ADC 24:110-115

79. Salvi S (2001) Pollution and allergic airways disease. Curr Opin Allergy Clin Immunol 1:35-41

80. Schmitz R, Atzpodien K, Schlaud M (2012) Prevalence and risk factors of atopic diseases in German children and adolescents. Pediatric allergy and immunology: official publication of the European Society of Pediatric Allergy and Immunology 23:716-723

81. Schnass W, Hüls A, Vierkötter A, Krämer U, Krutmann J, Schikowski T (2018) Traffic-related air pollution and eczema in the elderly: Findings from the SALIA cohort. Int J Hyg Environ Health 221:861-867

82. Schraufnagel DE, Balmes JR, Cowl CT et al (2019) Air Pollution and Noncommunicable Diseases: A Review by the Forum of International Respiratory Societies' Environmental Committee, Part 2: Air Pollution and Organ Systems. Chest 155:417-426

83. Tang KT, Ku KC, Chen DY, Lin CH, Tsuang BJ, Chen YH (2017) Adult atopic dermatitis and exposure to air pollutants-a nationwide population-based study. Annals of allergy, asthma \& immunology: official publication of the American College of Allergy, Asthma, \& Immunology 118, 351-355

84. Tecer LH, Alagha O, Karaca F, Tuncel G, Eldes N (2008) Particulate matter (PM(2.5), PM(10-2.5), and PM(10)) and children's hospital admissions for asthma and respiratory diseases: a bidirectional case-crossover study. J Toxicol Environ Health A 71:512-520

85. Teng B, Zhang X, Yi C, Zhang Y, Ye S, Wang Y, Tong DQ, Lu B (2017) The Association between Ambient Air Pollution and Allergic Rhinitis: Further Epidemiological Evidence from Changchun, Northeastern China. Int J Environ Res Public Health 14

86. To T, Zhu J, Stieb D, Gray N et al (2020) Early life exposure to air pollution and incidence of childhood asthma, allergic rhinitis and eczema. The European respiratory journal 55

87. Wang A, An YF, Zhao CQ (2008) Effects of sulphur dioxide inhalation on allergic rhinitis in mice. Chinese journal of otorhinolaryngology head and neck surgery 43:509-513

88. Wang IJ, Tung TH, Tang CS, Zhao ZH (2016) Allergens, air pollutants, and childhood allergic diseases. Int J Hyg Environ Health 219:66-71

89. Wang J, Zhang Y, Li B et al (2021) Asthma and allergic rhinitis among young parents in China in relation to outdoor air pollution, climate and home environment. Sci Total Environ 751:141734

90. Wang M, Wang S, Wang X et al (2020) The association between PM(2.5) exposure and daily outpatient visits for allergic rhinitis: evidence from a seriously air-polluted environment. Int J Biometeorol 64:139-144

91. Wang XW, Tian YH, Cao YY et al (2019) Association between Fine Particulate Air Pollution and Outpatient Visits for Eczema in Beijing, China: A Timeseries Analysis. Biomedical and environmental sciences: BES 32, 624-627

92. Wood HE, Marlin N, Mudway IS et al (2015) Effects of Air Pollution and the Introduction of the London Low Emission Zone on the Prevalence of Respiratory and Allergic Symptoms in Schoolchildren in East London: A Sequential Cross-Sectional Study.PloS one10, e0109121

93. Wu Y et al (2021) Air pollution and DNA methylation in adults: A systematic review and meta-analysis of observational studies. Environmental pollution (Barking, Essex: 1987) 284, 117152

94. Yang BY, Qian Z, Howard SW, Vaughn MG, Fan SJ, Liu KK, Dong GH (2018) Global association between ambient air pollution and blood pressure: A systematic review and meta-analysis. Environmental pollution (Barking, Essex: 1987) 235, 576-588

Page 18/19 
95. Zhang F, Wang W, Lv J, Krafft T, Xu J (2011) Time-series studies on air pollution and daily outpatient visits for allergic rhinitis in Beijing, China. Sci Total Environ 409:2486-2492

96. Zhang H, Zhang X, Wang Q, Xu Y, Feng Y, Yu Z, Huang C (2021a) Ambient air pollution and stillbirth: An updated systematic review and meta-analysis of epidemiological studies. Environmental pollution (Barking, Essex: 1987) 278, 116752

97. Zhang L, Jing D, Lu Q, Shen S (2021b) NO(2) exposure increases eczema outpatient visits in Guangzhou, China: an indication for hospital management. BMC Public Health 21:506

98. Zhou PE, Qian ZM, McMillin SE, Vaughn MG, Xie ZY, Xu YJ, Lin LZ, Hu LW, Yang BY, Zeng XW, Zhang WJ, Liu RQ, Chen G, Dong GH (2021) Relationships between Long-Term Ozone Exposure and Allergic Rhinitis and Bronchitic Symptoms in Chinese Children. Toxics 9

99. Zou QY, Shen Y, Ke X, Hong SL, Kang HY (2018) Exposure to air pollution and risk of prevalence of childhood allergic rhinitis: A meta-analysis. Int J Pediatr Otorhinolaryngol 112:82-90

\section{Figures}

\section{Figure 1}

The process of articles selection

\section{Figure 2}

Forest plot of subgroup analysis for diseases

\section{Figure 3}

Mechanistic insight into air pollutants and IgE mediated allergic diseases

Nod 1: nucleotide-binding oligomerization domain-containing protein 1; NF-KB: nuclear factor (NF)-KB; EOS: eosinophils; AHR: aromatic hydrocarbon receptor.

\section{Supplementary Files}

This is a list of supplementary files associated with this preprint. Click to download.

- supplementarymaterial.docx 